\title{
Extracellular vesicles from skin precursor-derived Schwann cells promote axonal outgrowth and regeneration of motoneurons via Akt/mTOR/p70S6K pathway
}

\author{
Xia Wu ${ }^{1 \#}$, Liting Wang ${ }^{2 \#}$, Meng Cong ${ }^{1}$, Mi Shen $^{1}$, Qianru He ${ }^{1}$, Fei Ding ${ }^{1,3}$, Haiyan Shi ${ }^{1,2} \wedge$ \\ ${ }^{1}$ Key Laboratory of Neuroregeneration of Jiangsu and Ministry of Education and Co-innovation Center of Neuroregeneration, Nantong University, \\ Nantong, China; ${ }^{2}$ Department of Pathophysiology, School of Medicine, Nantong University, Nantong, China; ${ }^{3}$ Jiangsu Clinical Medicine Center of \\ Tissue Engineering and Nerve Injury Repair, Affiliated Hospital of Nantong University, Nantong, China \\ Contributions: (I) Conception and design: X Wu, H Shi; (II) Administrative support: F Ding; (III) Provision of study materials or patients: None; \\ (IV) Collection and assembly of data: X Wu, L Wang, M Cong, M Shen, Q He; (V) Data analysis and interpretation: X Wu, H Shi; (VI) Manuscript \\ writing: All authors; (VII) Final approval of manuscript: All authors. \\ \#These authors contributed equally to this work. \\ Correspondence to: Dr. Haiyan Shi; Prof. Fei Ding. Key Laboratory of Neuroregeneration of Jiangsu and Ministry of Education and Co-innovation \\ Center of Neuroregeneration, Nantong University, 19 Qixiu Road, Nantong, China. Email: haiyansh@ntu.edu.cn; dingfei@ntu.edu.cn.
}

Background: Skin precursor-derived Schwann cells (SKP-SCs) have been shown to benefit the recovery of spinal cord injury (SCI) and peripheral nerve injury (PNI) with motor dysfunction. However, the effect of extracellular vesicles (EVs) from SKP-SCs responsible for neuroregeneration remains unknown.

Methods: Based on the obtainment and identification of rat SKP-SCs and their derived EVs, the primary rat injury model of motoneurons resulting from axotomy in vitro or nerve crush in vivo, as well as the secondary rat ischemic hypoxic injury model of motoneuron exposure to oxygen-glucose-deprivation (OGD) in vitro, were treated with EVs from skin precursor-derived Schwann cells (SKP-SC-EVs), respectively. Then, the axonal outgrowth and regrowth was observed and compared, and cell viability as well as the protein kinase B/mammalian target of rapamycin/p70 S6 kinase (Akt/mTOR/p70S6K) signaling pathway was detected, moreover, rapamycin (an mTOR inhibitor) was used to further reveal the underlying molecular mechanism.

Results: The internalization of SKP-SC-EVs by neuronal cells was identified in vitro and in vivo. Besides the pro-axonal outgrowth effect of SKP-SC-EVs, prospectively, the treatment of OGD-injured motoneurons with SKP-SC-EVs potentiated the restoration of neuronal viability and axonal regrowth. Furthermore, the axotomizing injury could be improved with SKP-SC-EVs treatment in vitro and in vivo. Finally, it was shown that the application of SKP-SC-EVs could activate the Akt/mTOR/p70S6K signaling pathway that can be abolished by rapamycin.

Conclusions: In summary, the addition of SKP-SC-EVs could regulate the cell growth and death signaling pathway mediated by Akt/mTOR/p70S6K, owing to the transmission of cargos in EVs to damaged motoneurons, which leads to axonal regrowth and neuronal resurrection. Thus, SKP-SC-EVs treatment could be a novel promising strategy for improving the axonal outgrowth and regeneration of motoneurons.

Keywords: Skin precursor-derived Schwann cells (SKP-SCs); motoneurons; extracellular vesicles (EVs); axonal regeneration; Akt/mTOR/p70S6K

Submitted Sep 08, 2020. Accepted for publication Nov 28, 2020.

doi: $10.21037 / \mathrm{atm}-20-5965$

View this article at: http://dx.doi.org/10.21037/atm-20-5965

^ ORCID: 0000-0002-9615-1270. 


\section{Introduction}

In recent years, accumulating evidence has suggested that extracellular vesicles (EVs) derived from therapeutic cells can potentiate tissue regeneration, participate in a wide range of biological processes, and function as potential alternatives to stem cell therapy (1). Nonetheless, seeking approaches for neuroregeneration is still tremendously challenging, especially in the case of locomotor dysfunction caused by a traumatic brain injury or spinal cord injury (SCI), where patients are facing a poor quality of life and overburdening their family and society (2).

When exploiting an EVs therapeutic potential, the first consideration is the parent cell source and the recipient cells or tissues; the phenotype and composition of EV populations reflects the properties of donor cells and may also influence their impact on target cells and tissues, although the specialties are still unclear $(3,4)$. The highly specialized nature of EVs as nanoscale messengers that deliver biological signals and thus mediate intercellular communication, dictates that within populations of $\mathrm{EVs}$, important properties including morphology, membrane composition, and content vary substantially; this heterogeneity arises in response to the nature, state, and environmental conditions of the cell source $(5,6)$.

Currently, multiple efforts are underway in developing mesenchymal stem/stromal cells (MSCs)-derived EVs as therapeutic agents $(7,8)$. Besides MSCs, a number of other cell types with stem-cell-like properties are associated with neuroprotective and neuroreparative potential, and can also be harnessed for therapeutic applications. Furthermore, the neuroregeneration process is implicated with multiple types of cells in the tissue microenvironment, and different types of cells in various states exert respective effect on the target recipient cells. For instance, when migrating Schwann cells (SCs) direct axon regeneration within the peripheral nerve bridge, they not only migrate into the area of damaged tissue and become a key component of regenerating tissue, but also secrete signaling molecules to attract macrophages, support neuronal survival, promote axonal regrowth, activate local MSCs, and interact with other cell types (9). In addition, it was recently reported that muscle-derived EVs could influence motoneuron regeneration accuracy (10), and small EVs derived from M2 bone marrow-derived macrophages could promote motor function recovery in SCI (11).

Given the poor regenerating potential of motoneurons in the nervous system, and the spontaneous support provided by SCs for axonal regeneration in the peripheral nervous system, we were induced to exploit the potential of SCderived EVs. In recent years, SCs derived from adult neural crest stem/progenitor cells, including skin precursors (SKPs), were applied to repair traumatic and degenerative neurological diseases and some progress has been made, qualifying them as an important tool in neuroregenerative medicine (12). Nevertheless, contrary to common belief, increasing publications have implied that cell replacement is not the primary mechanism by which these cells exert their therapeutic potential. It became evident that transplanted cells exploit released EVs as a paracrine communication mechanism to augment endogenous neuroprotection and improve neurological impairment (13). In regards to MSCderived $\mathrm{EV}$-based therapies exerting regenerative effects on neurological impairment, the mechanisms were involved in neurogenesis, neuronal proliferation, neuronal survival, neurogenesis, and angiogenesis $(13,14)$. Additionally, primarily cultured SCs could release exosomes to repair rat sciatic nerve injury through Rho inhibition (15); likewise, SC-like differentiated adipose stem cells (ADSCs) can promote neurite outgrowth of neurons via secreted exosomes and RNA transfer (16), which suggests parallels between the 'SCs' and 'ADSC derived SCs' mode of actions.

In particular, SKP-derived SCs (SKP-SCs) were recently demonstrated to improve the behavioral recovery in rats with acute and delayed peripheral nerve injury (PNI) (17); furthermore, SCs raised from neonatal SKPs can improve functional recovery after acute transplantation into the partially injured rat cervical spinal cord (18). Hence, these findings indicated that EVs from skin precursorderived Schwann cell (SKP-SC-EVs) can be postulated as a promising candidate $\mathrm{EV}$ population for the treatment of motoneuron impairment, as a cell-free therapeutic approach to neuroregeneration. Therefore, our interest was elicited as to whether and how SKP-SC-EVs would act as important mediators of intercellular communication to exert their therapeutic benefit to damaged motoneurons.

Furthermore, the pathophysiological alteration of damaged motoneurons in SCI is characterized with primary and secondary injury. The primary injury initiated by mechanical trauma includes traction and compression forces, and simultaneously, blood vessels are damaged, axons disrupted, and neural-cell membranes broken (19). Thereby, the initial mechanical trauma is followed by a significant secondary injury which includes local ischemia, 
pro-apoptotic signaling, release of cytotoxic factors, and inflammatory cell infiltration $(20,21)$. Accordingly, to investigate the therapeutic effect of SKP-SC-EVs, we established models to simulate disrupted axons, including crush injury of rat sciatic nerves in vivo and axotomizing injury of motoneurons in vitro; besides, neuronal injury model exposure to oxygen-glucose-deprivation (OGD) culture condition was established to simulate the ischemic hypoxic damage of motoneurons.

In the present work, we obtained and expanded rat SKPSCs in vitro before isolating EVs from the culture medium, then constructed a cell-free therapy research platform based on SKP-SC-EVs. we applied SKP-SC-EVs to the damaged motoneurons and nerves, then observed and evaluated the axonal outgrowth and regrowth in different models. Meanwhile, to explore the underlying mechanism of proaxonal regrowth involving with SKP-SC-EVs, we detected the activation of protein kinase $\mathrm{B} / \mathrm{mammalian}$ target of rapamycin/p70 S6 kinase (Akt/mTOR/p70S6K) signaling pathway, which might be the potential targets of SKP-SCEVs on promoting the axonal regeneration of motoneurons. We present the following article in accordance with the ARRIVE reporting checklist (available at http://dx.doi. org/10.21037/atm-20-5965).

\section{Methods}

\section{Animals}

Sprague-Dawley (SD) rats were supplied by the Experimental Animal Center of Nantong University. The study was approved by the Administration Committee of Experimental Animals, Jiangsu Province, China. The study protocols involving animals were performed according to the Chinese Guidelines for the Care and Use of Laboratory Animals.

\section{Culture of SKPs and differentiation towards SCs}

Rats at postnatal day 1 were used for primary culture of SKPs as described previously (22). Briefly, back skin samples from neonatal SD rats were cut into $1-2 \mathrm{~mm}^{2}$ pieces, followed by careful removal of tissue underlying the dermis. Skin tissue was digested with $0.1 \%$ trypsin or $1 \mathrm{mg} / \mathrm{mL}$ collagenase for $45-60 \mathrm{~min}$ at $37^{\circ} \mathrm{C}$, then mechanically dissociated, followed by filtration through a $40 \mu \mathrm{m}$ cell strainer. Cells were plated at a density of $(1-2.5) \times 10^{4}$ cells $/ \mathrm{ml}$ in Dulbecco's modified eagle medium (DMEM)/F12 medium (Corning Cellgro, Manassas, VA, USA) containing $0.1 \%$ penicillin/streptomycin (Beyotime, Shanghai, China), $40 \mathrm{ng} / \mathrm{mL}$ fibroblast growth factor 2 (FGF2; R\&D System, Minneapolis, MN, USA), $20 \mathrm{ng} / \mathrm{mL}$ epidermal growth factor (EGF; R\&D), and 2\% B27 supplement (Gibco, Brooklyn, NY, USA), with 1-2 $\mathrm{mL}$ medium containing sufficient supplements added every 3 days.

For differentiation towards SCs, SKP spheres were dissociated and cultured on plates coated with poly-D-lysine (PDL; Sigma-Aldrich, St. Louis, MO, USA) and laminin (Corning, Brooklyn, NY, USA) in SKP-SC differentiation medium I, namely DMEM/F12 medium containing $0.1 \%$ penicillin/streptomycin, $40 \mathrm{ng} / \mathrm{mL}$ FGF2, $20 \mathrm{ng} / \mathrm{mL}$ EGF, $2 \%$ B27 supplement, and 5-10\% fetal bovine serum (FBS; Gibco, Carlsbad, CA, USA). After 3 days, the medium was changed to SC differentiation medium II, namely DMEM/ F12 (3:1) containing $0.1 \%$ penicillin/streptomycin, $5 \mu \mathrm{m}$ forskolin (Sigma-Aldrich, St Louis, MO, USA), $50 \mathrm{ng} / \mathrm{mL}$ heregulin-1 $\beta$ (R\&D), 2\% N2 supplement (StemCell Technologies, Vancouver, BC, Canada), and 1-5\% FBS. Once the proliferating SCs showed the identifiable morphology, the cell colonies were isolated and cultured in SC proliferation medium (the same as SC differentiation medium II) for passage culture.

\section{Isolation and detection of SKP-SC-EVs}

Firstly, we isolated the EVs released from SKP-SCs. The SKP-SCs were incubated in the proliferation medium without FBS for 2 days at about $80 \%$ confluence (cell density). The harvested conditioned culture medium was centrifuged at $500 \mathrm{~g}$ for $10 \mathrm{~min}$, followed by collection of the supernatants, and the purification of SKP-SC-EVs was then performed following the manual instructions of the exoEasy Maxi Kit (Qiagen, Germantown, MD, USA).

Afterwards, the concentration and size distribution of SKP-SC-EVs were identified by nanoparticle tracking analyses (NTA, Particle Metrix, Ammersee, Germany), meanwhile, the morphology of EVs was observed under transmission electron microscopy (TEM, Hitachi, Tokyo, Japan).

\section{Culture of motoneurons and promoting of axonal outgrowth}

As previously described (23), a pregnant rat was sacrificed on day 13.5 of gestation by cervical dislocation. After 
disinfection, the fetal rats were removed and placed in Leibovitz's L-15 medium (Gibco, Life Technology, NY, USA). The spinal cord of fetal rats was separated, digested with $0.125 \%$ trypsin at $37{ }^{\circ} \mathrm{C}$ for $30 \mathrm{~min}$, and single cell suspension was obtained by mechanical dissociation, followed by purification of the motoneurons in $15 \%$ OptiPrep gradient centrifugation solution (Sigma-Aldrich, St. Louis, MO, USA). The obtained motoneurons were inoculated on culture apparatus coated with poly-l-lysine (PLL; Sigma-Aldrich, St. Louis, MO, USA), including coverslips, plates, and dishes, with neurobasal motoneuron culture medium (Gibco, Life Technology, NY, USA) containing 2\% B27, 1\% glutamax (Gibco, Carlsbad, CA, USA), and $1 \%$ penicillin/streptomycin. The further experiments were conducted following the steps outlined below.

To examine the effect of SKP-SC-EVs on promoting the axonal outgrowth of motoneurons, EVs were added into the culture medium at $4 \mathrm{~h}$ after motoneuron inoculation, then the axonal outgrowth in culture dishes was observed at 12 , 24 , and $36 \mathrm{~h}$ after treatment with different concentrations of EVs $\left(0.5 \times 10^{8}, 1 \times 10^{8}, 2 \times 10^{8}, 4 \times 10^{8}\right.$, and $8 \times 10^{8}$ particles $\left./ \mathrm{mL}\right)$. Afterwards, the axonal outgrowth of motoneurons in a microfluidic device was observed at $72 \mathrm{~h}$ after EV treatment daily, at a preferred concentration. Finally, the axonal length and branches of $~ 100$ motoneurons were analyzed by Image J software (Version 1.8.0; National Institutes of Health, USA; https://imagej.net/Downloads) after immunofluorescence staining.

\section{Immunofluorescence staining}

To identify the expression of cell protein markers, cell samples were fixed with $4 \%$ paraformaldehyde (PFA) (Beyotime, Shanghai, China) at room temperature (RT) for $15 \mathrm{~min}$ and then washed 3 times with phosphate buffer saline (PBS). Next, the cells were closed with the blocking solution (Beyotime) at RT for $1 \mathrm{~h}$, then incubated with the primary antibody at $4{ }^{\circ} \mathrm{C}$ overnight, followed by incubation with the secondary antibody at RT in the dark for $2 \mathrm{~h}$. Finally, cells were counterstained with 4',6-diamidino-2phenylindole (DAPI) (Sigma-Aldrich, MO, USA), and the photomicrographs were taken by confocal microscope (SP5, Leica, Mannheim, Germany).

The primary antibodies used included mouse anti-S100 $\beta$ (1:500, Thermo Fisher Scientific, Waltham, MA, USA), chicken anti-glial fibrillary acidic protein (GFAP; 1:1,000, Abcam, Cambridge, MA, USA), mouse anti- $\beta$-tubulin3
(TUJ1) (1:400, Abcam), rabbit anti-neurofilament 200 (NF200; 1:400, Sigma-Aldrich), and chicken anti-choline acetyltransferase (ChAT, 1:200, Abcam). The fluorescentlabeled secondary antibodies used included goat anti rabbit immunoglobulin G (IgG) cyanine 3 (Cy3) (1:400, Abcam), donkey anti chicken IgG Fluorescein isothiocyanate (FITC) (1:400, Abcam), goat anti mouse IgG 594 (1:400, Thermo Fisher Scientific), and goat anti mouse IgG FITC (1:400, Abcam).

\section{OGD-injury of motoneurons}

To establish an OGD-injury model of motoneurons, 4 days after inoculation, the primary cultured motoneurons were switched to glucose-free neurobasal medium (Gibco) containing $2 \% \mathrm{~B} 27,1 \%$ glutamax, and $1 \%$ penicillin/ streptomycin, cultured in $37{ }^{\circ} \mathrm{C}$ incubator with $5 \% \mathrm{CO}_{2}$ and $1 \%$ oxygen concentration for $1 \mathrm{~h}$. Then, the cultures were switched back to their original culture condition with or without EVs. At $24 \mathrm{~h}$ after treatment, the morphological alteration of neurons was observed and photomicrographs were taken. After TUJ1 immunofluorescence staining, the average length of the longest neurites and the average branch numbers of neurites was assessed for $\sim 100$ motoneurons in each group.

\section{Cell viability assay}

To evaluate the cell viability of motoneurons, after the treatment with or without OGD and SKP-SC-EVs, cells were assessed with tetrazolium salt reduction assay by cell counting kit-8 (CCK8) (Dojindo, Kumomoto, Japan) assay. At $24 \mathrm{~h}$ after treatment with EVs, the CCK8 solution was added to each well in 96-well plates of different groups (6 wells per group). Prior to measuring the absorbance value at $450 \mathrm{~nm}$ by microplate reader, cells were incubated at $37^{\circ} \mathrm{C}$ for $2 \mathrm{~h}$. The results were expressed as the percentage of viability relative to the control.

\section{Axonal outgrowth, axotomy, and regrowth of motoneurons in microfluidic devices}

To further evaluate the effect of SKP-SC-EVs on axonal outgrowth in vitro, microfluidic devices with $150 \mu \mathrm{m}$ microgrooves (SND 150, Xona Microfluidics, Temecula, CA, USA) were assembled and prepared as described previously (24). Briefly, after ultraviolet irradiation sterilization, microfluidic devices were attached to the 
PLL-coated cover petri dish, then motoneurons were resuspended at $2 \times 10^{7}$ cells $/ \mathrm{mL}$ concentration in culture media and $10 \mu \mathrm{L}$ cell suspension was loaded into the soma chamber of a microfluidic device. Next, the model of axon transection of motoneurons in vitro was established as described previously; after $4 \mathrm{~d}$ of culturing motoneurons in regular medium, the axonal compartment was aspirated twice, each time lasted for 1-2 min, and the vacuum pressure for aspiration was $\geq 18$ inch- $\mathrm{Hg}$ to ensure the axon was completely transected (24). Then, PBS with or without EVs $\left(4 \times 10^{8}\right.$ particles $\left./ \mathrm{mL}\right)$ was added into chamber medium for $24 \mathrm{~h}$ before performing TUJ1 immunofluorescence staining, and images were taken under the inverted fluorescence microscope (DMi8, Leica, Mannheim, Germany). For both groups, the average length of the 15 longest axons was assessed.

\section{Nerve crush injury and treatment with SKP-SC-EVs}

The SD Rats (male, weight 180-200 g, n=9 per group) were randomized into control and treatment groups in laboratory. After anesthesia of rats by intraperitoneal injection with compound anesthetics (dose $=0.35 \mathrm{~mL} / 100 \mathrm{~g}$ ) containing $42.5 \mathrm{mg} / \mathrm{mL}$ chloral hydrate and $8.86 \mathrm{mg} / \mathrm{mL}$ pentobarbital sodium, the left sciatic nerve was exposed, then crushed for 30 seconds with toothless needle holding forceps (straight type, $13 \mathrm{~cm}$, Halsey, Boston, MA, USA). The crush site was sutured with surgical 10-0 nylon monofilament (Ethicon, Somerville, NJ, USA). Meanwhile, SKP-SC-EVs were resuspended in sterile $\mathrm{PBS}$, then $10 \mu \mathrm{L}$ volume $\mathrm{PBS}$ with or without $\mathrm{EV}$ particles (at concentration of $1 \times 10^{9} / \mu \mathrm{L}$ ) was injected into the nerve epineurium $3 \mathrm{~mm}$ distal to the crush site with a microsyringe $(0.5 \mathrm{~mm}$ needle size, $25 \mu \mathrm{L})$.

\section{Immunobistochemical analysis}

To analyze the efficacy of SKP-SC-EVs on axonal regeneration in vivo, 4 days after nerve crush, the left sciatic segments were separated after the euthanasia of rats, then nerves were fixed in $4 \%$ PFA for $12 \mathrm{~h}$, followed by gradient dehydration. After these steps had been carried out, longitudinal cryostat sections (10 $\mu \mathrm{m}$ thick) were made by freezing microtome (CM3050 S, Leica, Mannheim, Germany), then immunofluorescence stained by rabbit anti-growth-associated protein 43 (GAP43) polyclonal antibody (1:400, Abcam) overnight at $4{ }^{\circ} \mathrm{C}$, followed by incubation with secondary antibody Alexa Fluor 488-goat anti-rabbit IgG (1:600, Abcam) at RT in the dark for $2 \mathrm{~h}$.
Finally, samples were washed in PBS before being mounted. Following this, the fluorescence images of regenerated nerves were captured using a fluorescence microscope (Axio Imager M2, Zeiss, Oberkochen, Germany), and the length of regenerating nerve fibers was measured and evaluated.

\section{Internalization of PKH67-labeled SKP-SC-EV s by neural cells}

Firstly, the internalization of SKP-SC-EVs by the primarily cultured motoneurons was undertaken using vital dyes (PKH67) in vitro, followed by immunofluorescence staining and observation. Briefly, EVs were stained using PKH67 green fluorescent linker mini kit (Sigma-Aldrich, St. Louis, MO, USA), then the EVs suspension was concentrated by Amicon Ultra $10 \mathrm{kDa}$ tubes (Millipore, Billerica, MA, USA). Next, motoneurons were incubated with the PKH67labeled EVs or PKH67 vehicle for $4 \mathrm{~h}$, then motoneurons were washed with PBS and fixed with $4 \%$ PFA, followed by TUJ1 immunofluorescence staining and images taken under confocal microscopy.

Subsequently, for in vivo internalization experiments ( $n=4$ per group), SKP-SC-EVs were stained with PKH67 as above-mentioned, then microinjected into intact sciatic nerve epineurium in $10 \mu \mathrm{L}$ volume PBS, and PBS that incubated with PKH67 simultaneously was set as the vehicle control. At $24 \mathrm{~h}$ post-injection, nerve segments were separated, frozen, and cut into longitudinal and transverse cryostat sections ( $10 \mu \mathrm{m}$ thick), followed by immunofluorescence staining with rabbit anti-rat polyclonal antibody NF200 (1:500, Sigma-Aldrich) and secondary antibody incubation. Finally, photomicrographs were captured by confocal microscope.

\section{Western blot}

In order to detect whether the mTOR pathway was involved in the neuroreparative effect of SKP-SC-EVs, the primarily cultured motoneurons were divided into different groups, and treated with or without OGD, EVs, and rapamycin $(10 \mathrm{nM}$, Cell Signaling Technology, CST, Beverly, MA, USA). At $12 \mathrm{~h}$ after EVs treatment, the expression of AKT, p-Akt, mTOR, p-MTOR, p70S6K, and $\mathrm{p}-\mathrm{P} 70 \mathrm{~S} 6 \mathrm{~K}$ in motoneurons from different groups was analyzed by western blot. Briefly, cell extracts were obtained from motoneurons in respective groups using Pierce RIPA Buffer Lyse (Thermo Fisher Scientific, Waltham, MA, USA) containing $1 \mathrm{mM}$ phenylmethanesulfonyl fluoride 
(PMSF) (Sigma Chem. Co., St Louis, MO, USA) and protease and phosphatase inhibitors (CST, Danvers, MA, USA). The supernatant was centrifuged at $12,000 \mathrm{rpm}$ at $4{ }^{\circ} \mathrm{C}$ for $30 \mathrm{~min}$. The concentration of the protein was determined by a micro-bicinchoninic acid (BCA) assay kit (Thermo Fisher Scientific, Waltham, MA, USA). Equal amounts of total protein from every sample were separated by $10 \%$ or $12 \%$ sodium dodecyl sulfate-polyacrylamide gel electrophoresis (SDS-PAGE) and subsequently transferred to polyvinylidenedifluoride (PVDF) membranes (Millipore, Billerica, MA, USA). Then, membranes were incubated with the primary antibody respectively at $4{ }^{\circ} \mathrm{C}$ overnight, including phospho-mTOR (p-mTOR) (Ser2448) antibody (1:1,000, CST, Danvers, MA, USA), mTOR rabbit polyclonal antibody $(1: 1,000$, Proteintech, Rosemont, IL, USA), Akt polyclonal antibody (1:1,000, CST), phosphoAkt (p-Akt) (Ser473) antibody (1:1,000, CST), p70S6K (1:1,000, CST) antibody, and phospho-p70S6K (p-p70S6K) (Ser424) antibody (1:1,000, CST). After 3 washes with $1 \times$ tris-buffered saline with $0.1 \%$ Tween 20 detergent (TBST), the membranes were incubated with horseradish peroxidase (HRP)-labeled goat anti-mouse IgG (1:5,000, Santa Cruz Biotechnology, Dallas, TX, USA) and HRP-labeled chicken anti-rabbit IgG (1:5,000, Abcam, Cambridge, MA, USA) at RT for $1 \mathrm{~h}$. The signals of membranes were detected with enhanced chemiluminescence (ECL) reagent kits (Beyotime, Shanghai, China). Finally, the band intensities were analyzed with the Image J software.

In addition, western blot was performed to detect the expressions of SKP-SC-EVs associated marker proteins, several primary antibodies were applied, including heat shock protein 70 (HSP70; 1:1,000, Proteintech, Rosemont, IL, USA), CD63 (1:1,000, Abcam), CD9 (1:1,000, Abcam), CD81 (1:1,000, Sigma-Aldrich), and tumor susceptibility gene 101 (TSG101; 1:1,000, Proteintech).

\section{Statistical analysis}

The quantitative results from 3 independent experiments were statistically analyzed by GraphPad Prism software (Version 5; San Diego, CA, USA; https://www.graphpadprism.cn/), and the data were presented as mean \pm SEM. Comparisons between groups were performed with oneway analysis of variance (ANOVA) and Tukey multiple comparisons test or Student's $t$-test (when the comparison was between two groups).

\section{Results}

\section{Identification of SKP-SCs and motoneurons}

Our findings were in line with previous results, after the primary culture at $\sim 2-3$ weeks, SKPs grew as floating spheres with nice refractivity, that could be observed under phase-contrast microscope (Figure 1A). Furthermore, the morphology of differentiated SKPs toward SCs showed typical a bipolar spindle-shape with strong refractivity of the cell body, then after passaged culture, the SKP-SCs grew side by side along the longitudinal axis (Figure 1B), meanwhile, immunofluorescence staining results demonstrated the positive expression of SC associated marker proteins S100 $\beta$ and GFAP (Figure 1C).

As far as motoneurons were concerned, at $2 \mathrm{~d}$ after primary culture, neurons showed small round or elliptic somas with short neurites and a few branches; then at $4 \mathrm{~d}$, the axons elongated with more sprouting branches, and long neurites forming a dendritic network (Figure 1D). Immunofluorescence staining indicated that motoneurons positively expressed NF-200 and ChAT, and the purity of motoneurons was $>90 \%$ (data not shown) (Figure 1E).

\section{Characterization of EVs derived from SKP-SCs}

To identify SKP-SC-EVs, the morphology, particle size and concentration, and the expression of marker proteins were all assessed. The NTA showed that the peak diameter of the SKP-SC-EVs was about $137 \mathrm{~nm}$ (Figure 2A), and TEM image displayed their cup-shaped morphology typically. The diameter data were consistent with each other across 2 assessments (Figure 2B).

Besides, western blot analysis showed that EVs positively expressed CD9, CD63, CD81 (tetraspanins), TSG101 (a protein involved in multivesicular body biogenesis), and HSP70 (a stress response protein). In contrast to their released EVs, SKP-SCs showed negative expression of CD9 and CD81, and lower expression of CD63 and TSG101, with even higher expression of HSP70 (Figure 2C).

Additionally, we examined whether SKP-SC-EVs could be transferred to motoneurons. After incubation with $\mathrm{EVs}$ for $4 \mathrm{~h}$ in vitro, immunostaining images showed the intake of PKH67-labeled EVs by motoneurons; in particular, the green particles were highlighted inside both the soma cytoplasm and axons (Figure 2D), indicating the internalization of EVs by motoneurons as expected. 

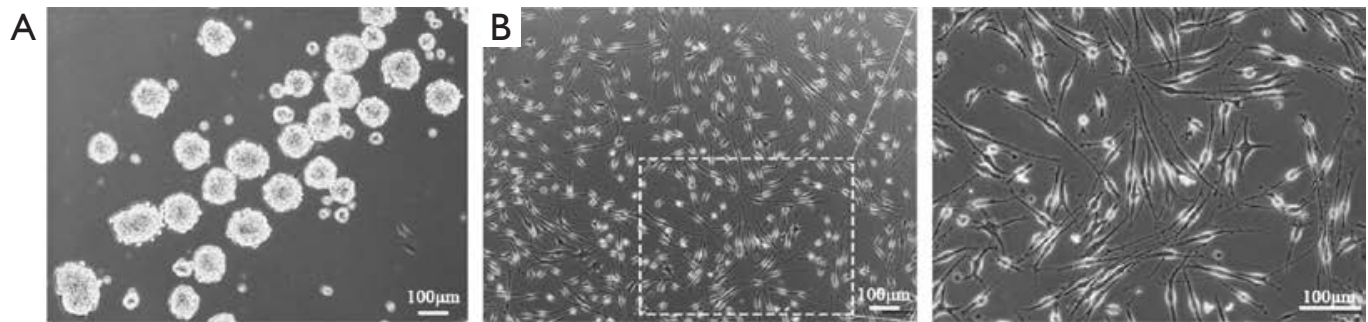

C
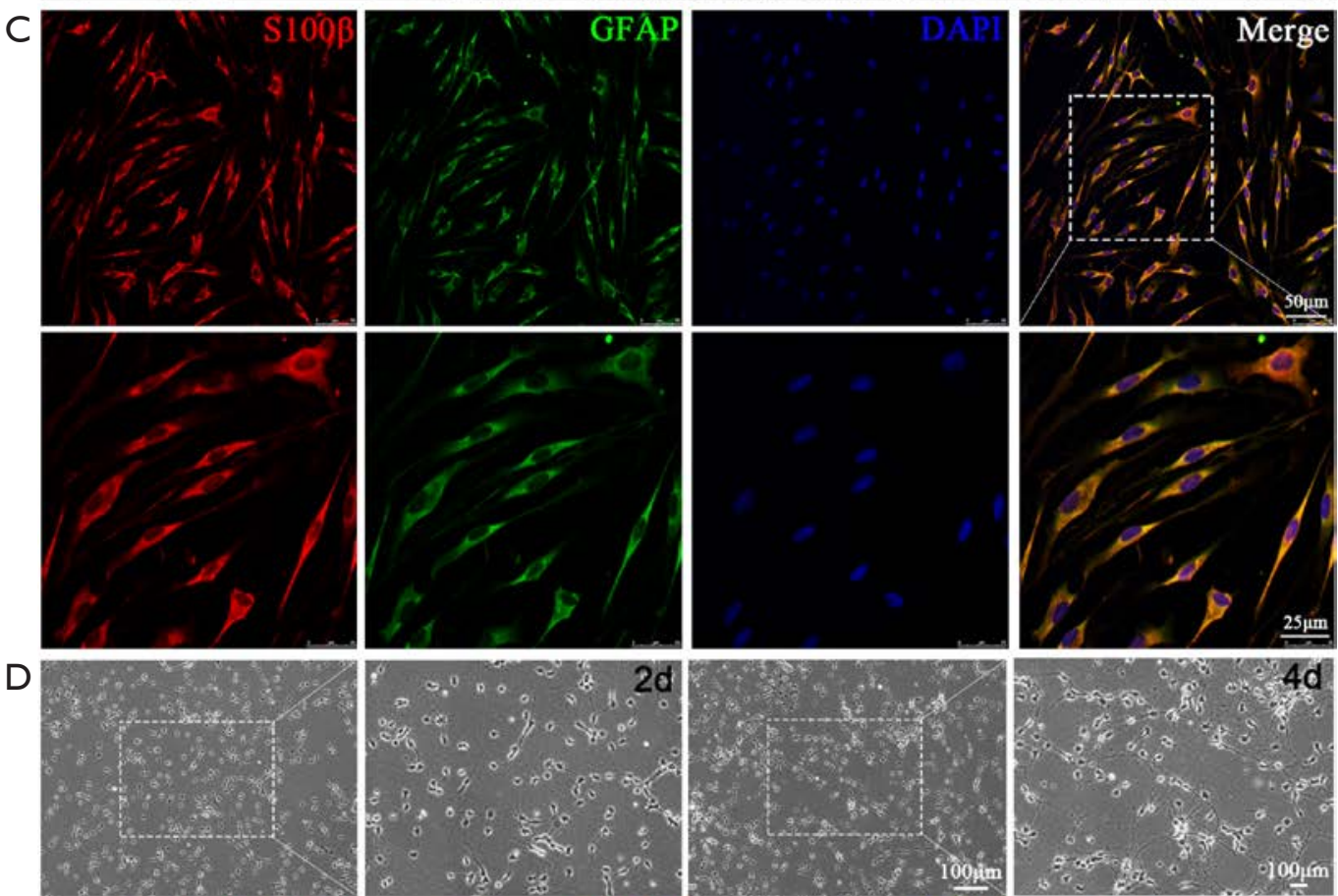

$\mathrm{E}$
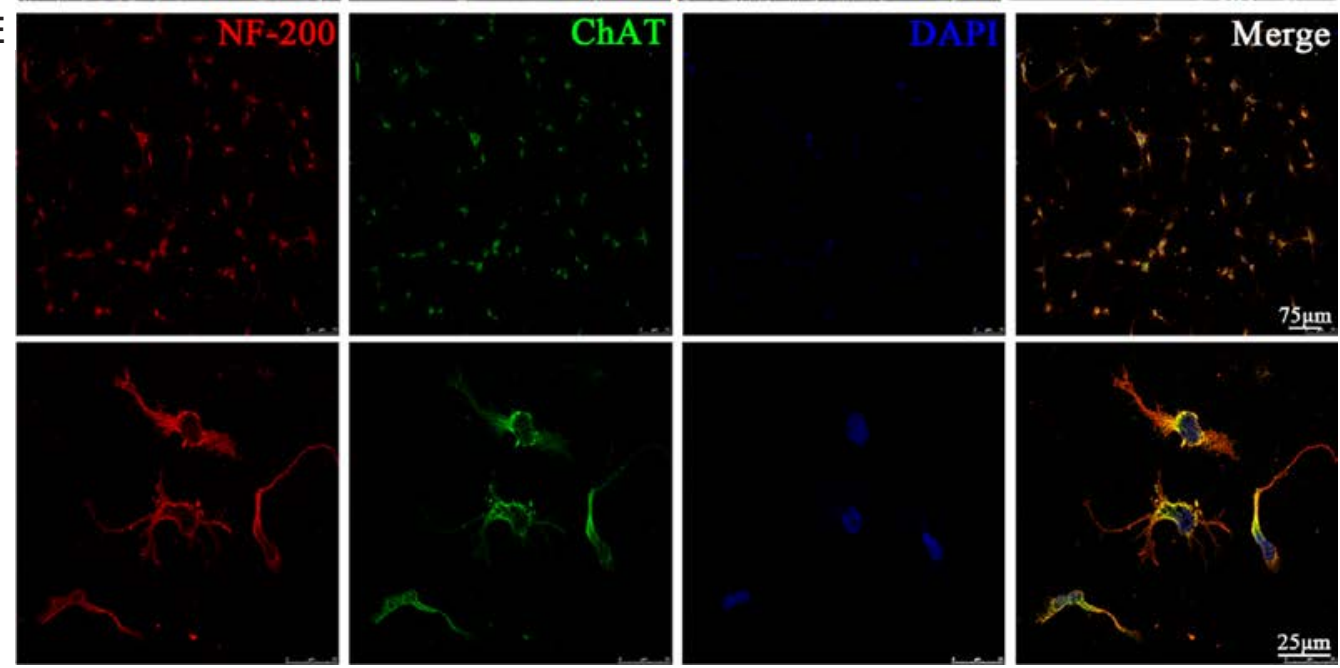

Figure 1 Identification of SKP-SCs and motoneurons. (A) Phase-contrast images showed the floating SKP spheres. Scale bar, $100 \mu \mathrm{m}$. (B) SKP-SCs showed a typical bipolar spindle-shape with side by side alignment. Scale bar, $100 \mu \mathrm{m}$. (C) Immunostained SKP-SCs positively expressed SC markers S100 $\beta$ (red) and GFAP (green) with DAPI stained cell nuclei (blue). Scale bar, $50 \mu \mathrm{m}, 25 \mu \mathrm{m}$. (D) Morphology of primarily cultured motoneurons with round somas and short neurites at day 2, and extended neurites forming a network at day 4. Scale bar, $100 \mu \mathrm{m}$. (E) Immunofluorescence stained motoneurons positively expressed NF-200 (red) and ChAT (green) with DAPI labeled nuclei (blue). Scale bar, $75 \mu \mathrm{m}, 25 \mu \mathrm{m}$. SKP, skin precursor; SC, Schwann cell; DAPI, 4',6-diamidino-2-phenylindole; GFAP, glial fibrillary acidic protein. 
A

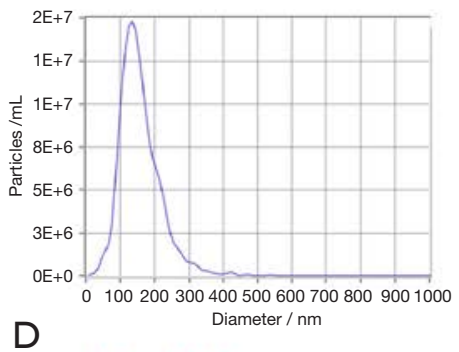

$\mathrm{D}$
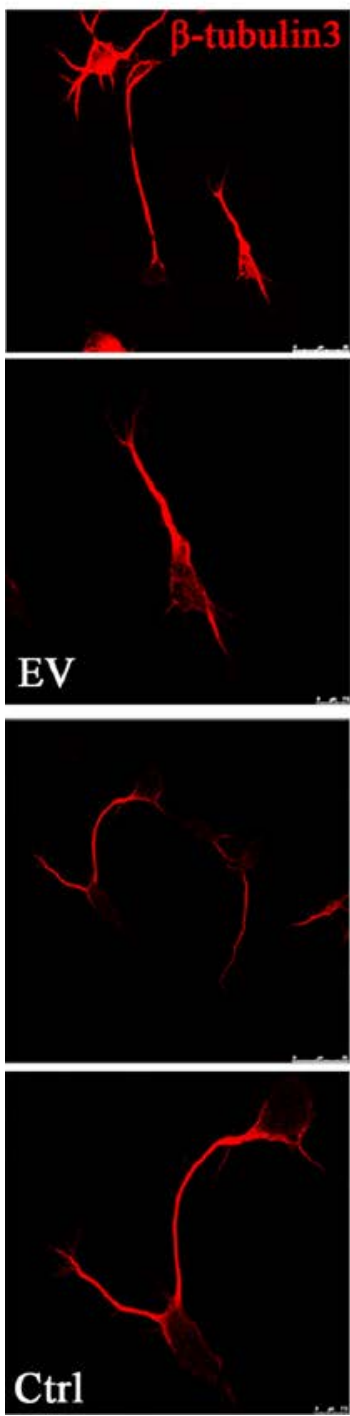

B
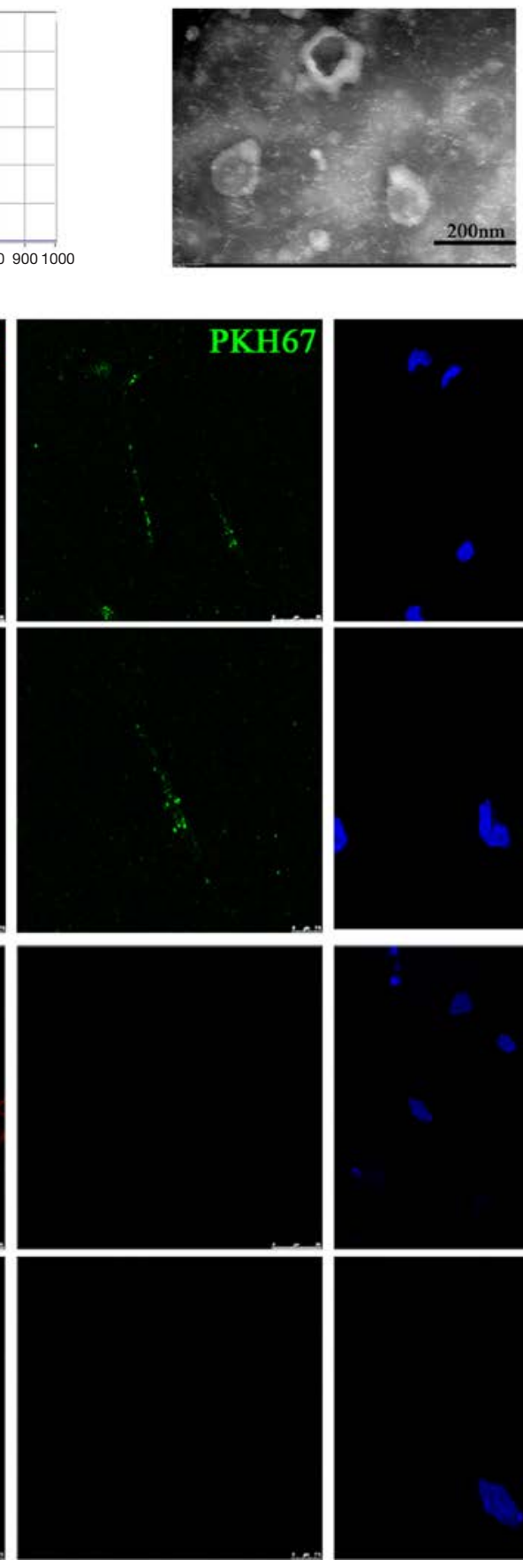

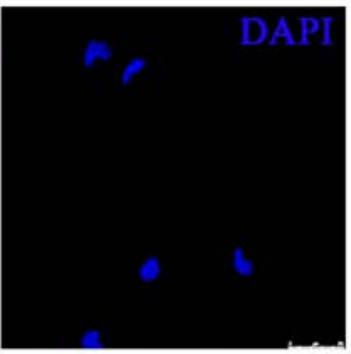

C
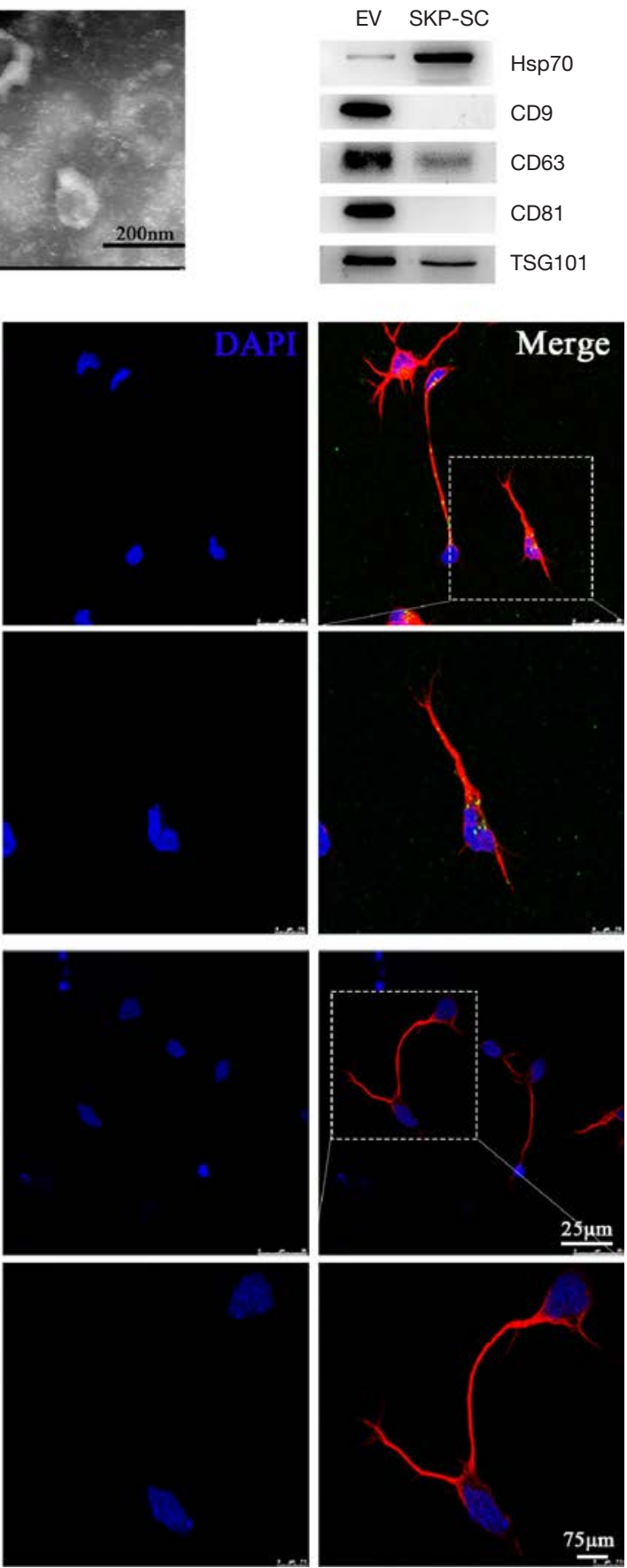

Figure 2 Characterization of EVs derived from SKP-SCs. (A) Representative size distribution of EVs derived from SKP-SCs detected by nanoparticle tracking analysis (NTA). (B) The typical cup-shape of EVs was showed under transmission electron microscopy (TEM). Scale bar, $200 \mathrm{~nm}$. (C) Representative western blot analysis showed the positive expression of CD9, CD63, CD81, HSP70, and TSG101 in SKPSC-EVs, SKP-SC was used as control. (D) Motoneurons were incubated with PKH67-labeled EVs or PBS (as control), photomicrographs showed the intake of PKH67-labeled EV particles (green) by TUJ1 stained motoneurons (red) with DAPI-stained nuclei (blue). Scale bar, $25 \mu \mathrm{m}$. The magnification image showed that the internalized EVs distributed in both soma of cytoplasm and axons of motoneurons. Scale bar, $75 \mu \mathrm{m}$. SKP, skin precursor; SC, Schwann cell; EV, extracellular vesicle; PBS, phosphate buffered saline; TUJ1, $\beta$-tubulin3; DAPI, 4',6-diamidino-2-phenylindole. 


\section{Axonal outgrowth promoting effect of SKP-SC-EVs}

To investigate whether SKP-SCs secreted EVs could affect axonal outgrowth, we assessed the axonal length change in 2 experiments. First, we evaluated the effects of different concentrations of EVs on motoneuron axonal outgrowth in regular culture plates; we observed neuronal morphological alteration at 3 time points (12, 24, and $36 \mathrm{~h}$ ), and the representative images with immunostaining of TUJ 1 at $24 \mathrm{~h}$ is shown in Figure $3 \mathrm{~A}$. After statistical analysis, we found that at each time point, when the concentration of EVs changed from $0.5 \times 10^{8}$ to $4 \times 10^{8}$ particles $/ \mathrm{mL}$, the axonal length was increasing significantly in a dose-dependent manner, but at the concentration of $8 \times 10^{8}$ particles $/ \mathrm{mL}$, the length of axons no longer increased; meanwhile, during the $36 \mathrm{~h}$ period, the positive influence of EVs showed a timedependent effect (Figure 3B,C,D). Therefore, in further experiments, the selected concentration of EVs was set at $4 \times 10^{8}$ particles $/ \mathrm{mL}$.

Subsequently, we further observed the effect of EVs on the axonal outgrowth of motoneurons by microfluidic devices, which compartmentalize the somas and axons of neurons (Figure 3E). Over 3 days, the SKP-SC-EVs $\left(4 \times 10^{8}\right.$ particles $\left./ \mathrm{mL}\right)$ or vehicle solutions (PBS as control) were supplemented on a daily basis. Prospectively, EVs treatment significantly augmented the length of axons compared with vehicle treatment (Figure $3 F, G$ ), showing that SKP-SC-EVs could stimulate axonal outgrowth of motoneurons in vitro.

\section{Reparative effect of SKP-SC-EVs on OGD-injured motoneurons}

To determine whether SKP-SC-EVs could promote neuronal recovery and axonal repair in injured motoneurons, we began by establishing a neuronal OGDinjury model. We found that OGD induced neuronal death and axonal disruption, and the pathological alteration was significantly reversed after treatment with EVs at the concentration of $4 \times 10^{8}$ particles $/ \mathrm{mL}$ (Figure $4 A$ ). We then measured the average length of the longest neurites and the average number of primary neurites of motoneurons; both quantitative indexes were significantly reduced in the OGD group compared with those in the control group, and they were significantly reversed in OGD + EV group compared with those in OGD group (Figure 4B,C).

In addition, we performed CCK 8 assay, and found that the cell viability of motoneurons decreased to
$58.7 \%$ after OGD, while it was significantly elevated in a dose-dependent manner after treatment with different concentrations of EVs. When EV concentration reached $16 \times 10^{8}$ particles $/ \mathrm{mL}$, the cell viability was equivalent to the control group. Interestingly, as the EV concentration continued to increase, the cell viability was higher in $32 \times 10^{8}$ particles $/ \mathrm{mL}$ concentration group than that in the control group (Figure 4D). Suggestively, SKP-SC-EVs showed a reparative effect on OGD-injured motoneurons.

\section{Axonal regrowth promoting effect of SKP-SC-EVs on axotomizing injury of motoneurons}

Since the primary injury of motoneurons in SCI and PNI usually results from mechanical damage, we utilized a microfluidic device to evaluate the effect of SKP-SC-EVs on axonal regrowth after axotomizing injury (Figure $5 A$ ). After loading motoneurons into the soma side compartment (left side) of the microfluidic device, most of the neuronal processes crossed the microgrooves and extended into the axon side compartment (right side) within 4 days (Figure $5 B$, up). Then, we performed the mechanical transection of axons by vacuum pump, and the axon retraction could be observed under the phase-contrast microscope (Figure 5B, bottom). Following this, the motoneurons were subjected to SKP-SC-EVs or vehicle (PBS as control) treatment. Intriguingly, the axon regeneration of motoneurons treated with EVs was notable with remarkable speed; 24 h later, axons were TUJ1 immunofluorescence stained, and the statistical results showed that the average axonal length of motoneurons in the $\mathrm{EV}$ treatment group was 1.5 times longer than that in the control group (Figure $5 C, D$ ).

\section{Pro-regenerative effect of SKP-SC-EVs on crushed nerve}

We further verified the pro-axonal regrowth effect of SKPSC-EVs using an in vivo model. Prior to evaluating the effect of SKP-SC-EVs on a crushed nerve, we examined whether SKP-SC-EVs could be internalized in nerves. At $24 \mathrm{~h}$ after local microinjection of PKH67-labeled EVs, the defined sciatic nerve segment was harvested and the cryostat sections were subjected to NF200 immunofluorescence staining. Results showed the distribution of green EVs particles on both longitudinal and cross sections of the nerve tissue, obviously, the longitudinal section displayed the co-localization of EVs with the nerve fibers, and the cross section also displayed an extensive distribution of EVs in the epineurium (Figure 6A). 
A
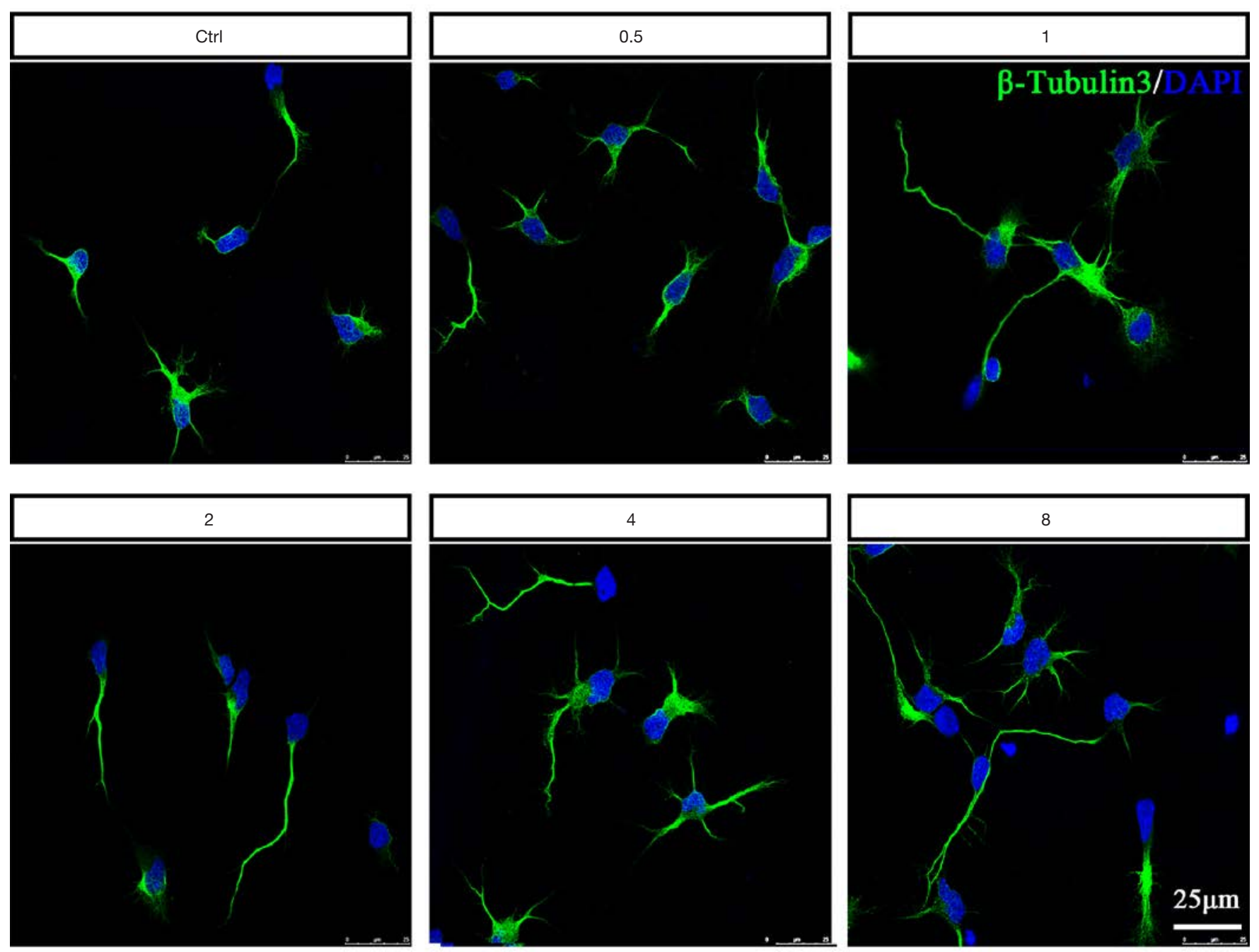

B

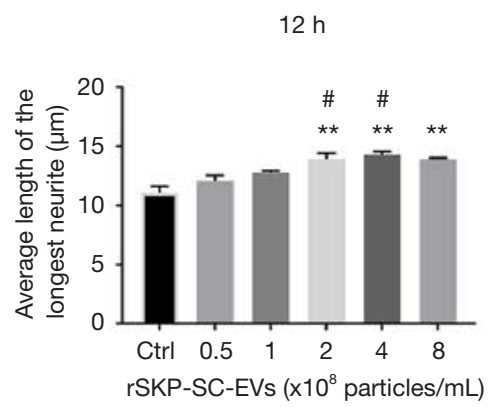

C

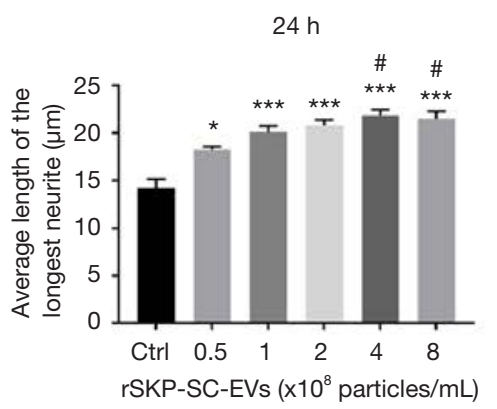

D

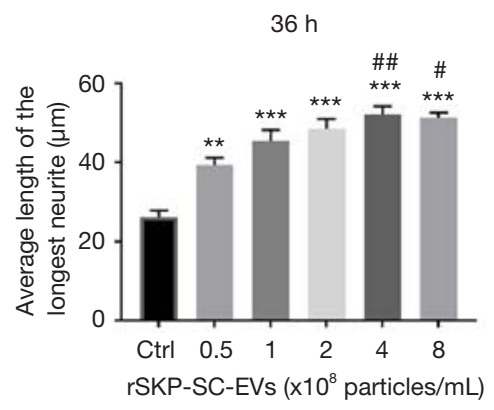

$25 \mu \mathrm{m}$

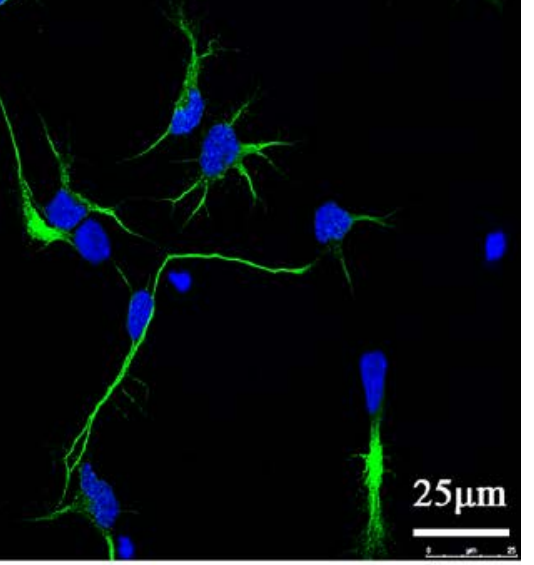

SKP-SC-EVs (x10 ${ }^{8}$ particles $\left./ \mathrm{mL}\right)$ 
$E$

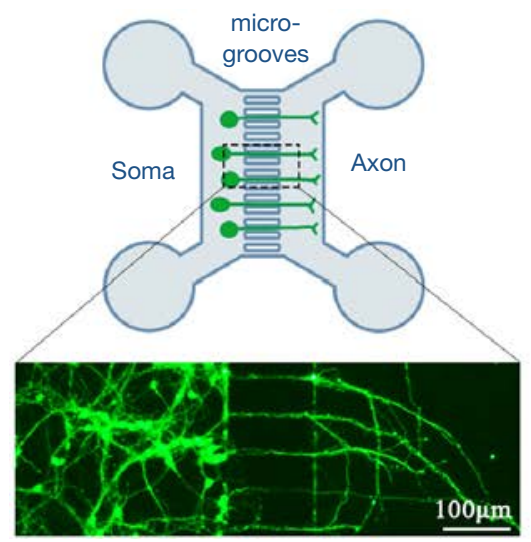

$\mathrm{F}$

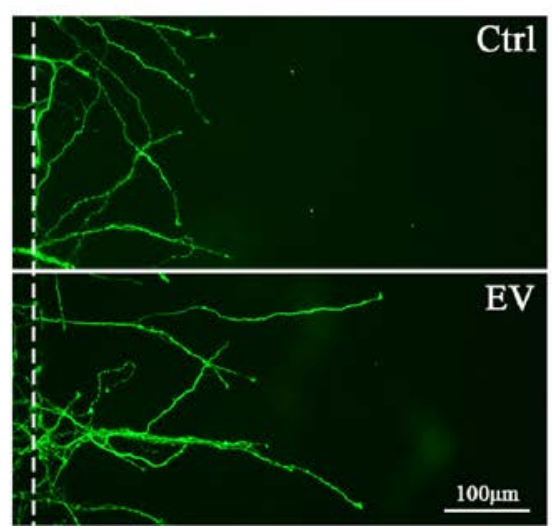

G

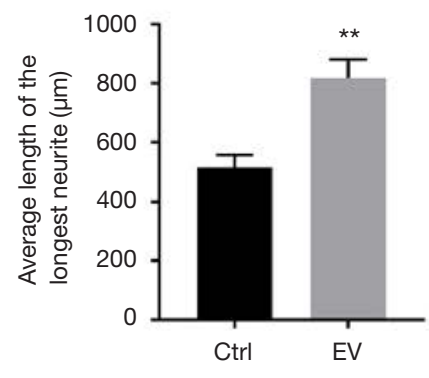

Figure 3 Axonal outgrowth promoting effect of SKP-SC-EVs. (A) Representative images of each group showed the morphology of motoneurons co-cultured with different concentration of EVs $\left(0.5 \times 10^{8}, 1 \times 10^{8}, 2 \times 10^{8}, 4 \times 10^{8}\right.$, or $8 \times 10^{8}$ particles $\left./ \mathrm{mL}\right)$ for $24 \mathrm{~h}$, displaying TUJ1-labeled green motor neurons and DAPI-stained blue nuclei. Scale bar, $25 \mu \mathrm{m}$. (B,C,D) Statistical histograms showed the increasing of the average length of the longest neurite in a dose-dependent manner at 12, 24, and $36 \mathrm{~h}$, respectively. For each group and experiment, $\sim 100$ neurons per condition were assessed. Mean $\pm \mathrm{SEM}, \mathrm{n}=3 ;{ }^{*}, \mathrm{P}<0.05,{ }^{* *}, \mathrm{P}<0.01,{ }^{* * *}, \mathrm{P}<0.001$, as compared with control group;, $\mathrm{P}<0.05$,

\#\#, $\mathrm{P}<0.01$, as compared with $0.5 \times 10^{8}$ particles/mL EV group. (E) Schematic of motoneurons cultured in a microfluidic device. Primary motoneurons were loaded in the soma chamber side, and axons traversed through the microgrooves, extending into the axon chamber side. Immunofluorescence photomicrograph showed TUJ1 stained motoneurons (green). Scale bar, $100 \mu$ m. (F) Representative images of TUJ1 immunofluorescence stained axons of motoneurons (green) in 2 groups. Scale bar, $100 \mu \mathrm{m}$. (G) Statistical histograms showed the average 15 longest neurite length was longer in $\mathrm{EV}$ treatment group than that in the control group. Mean $\pm \mathrm{SEM}, \mathrm{n}=3,{ }^{* *}, \mathrm{P}<0.01$, as compared with control group. SKP, skin precursor; SC, Schwann cell; EV, extracellular vesicle; TUJ1, $\beta$-tubulin3; DAPI, 4',6-diamidino-2-phenylindole.

Subsequently, the treatment of SKP-SC-EVs on crushed sciatic nerves was performed in rats ( 9 animals per group). At $4 \mathrm{~d}$ after treatment, freezing cut section samples of nerves were prepared, followed by GAP43 immunofluorescence staining. The axonal regeneration was evaluated by measuring the length of the regenerating nerve segments; consequently, it was shown that the density of nerve fibers increased significantly in the SKP-SC-EVs injection group (Figure $6 B$ ); on average, the length of nerve regrowth was 1.4 times longer in the EVs injection group than that in vehicle injection group (Figure 6C). The results further strengthened our confidence in the pro-regenerative effect of SKP-SC-EVs on transected axons after SCI and PNI in vivo.

\section{Underlying mechanism of Akt/mTOR/p70S6K patbway activation}

To elucidate whether SKP-SC-EVs activates the Akt/ mTOR/p70S6K pathway in damaged motoneurons, we applied an OGD-injury model in vitro to resemble the motoneurons injury in ischemic hypoxic condition in vivo.
At $12 \mathrm{~h}$ after EVs treatment, the change of signaling molecules were analyzed by western blot, including Akt, p-Akt, mTOR, p-mTOR, p70S6K, and p-p70S6K (Figure $7 A$ ). We found that the expression of $\mathrm{p}-\mathrm{Akt}$, $\mathrm{p}-\mathrm{mTOR}$, and $\mathrm{p}-\mathrm{p} 70 \mathrm{~S} 6 \mathrm{~K}$ in the OGD + vehicle group was lightly increased compared with that in the control group (Figure $7 B, C, D$ ). Whereas, the expression of $\mathrm{p}$-Akt and p-mTOR in the OGD + EV group was significantly higher than that in OGD + vehicle group, with p-p70S6K showing an trend of increase (Figure $7 B, C, D$ ). Importantly, after adding rapamycin (an inhibitor of mTOR), the expression of p-Akt, p-mTOR, and p-p70S6K was significantly decreased (Figure $7 B, C, D$ ). In our view the results emphasized the validity of our model and revealed the activation of Akt/mTOR/p70S6K pathway by SKP-SC$\mathrm{EVs}$ in pro-regenerative effect exerting process.

\section{Discussion}

Developing best-practice models for the therapeutic use of EVs involves the consideration of several important aspects, including cell culture condition, yield of EVs, and the 
A
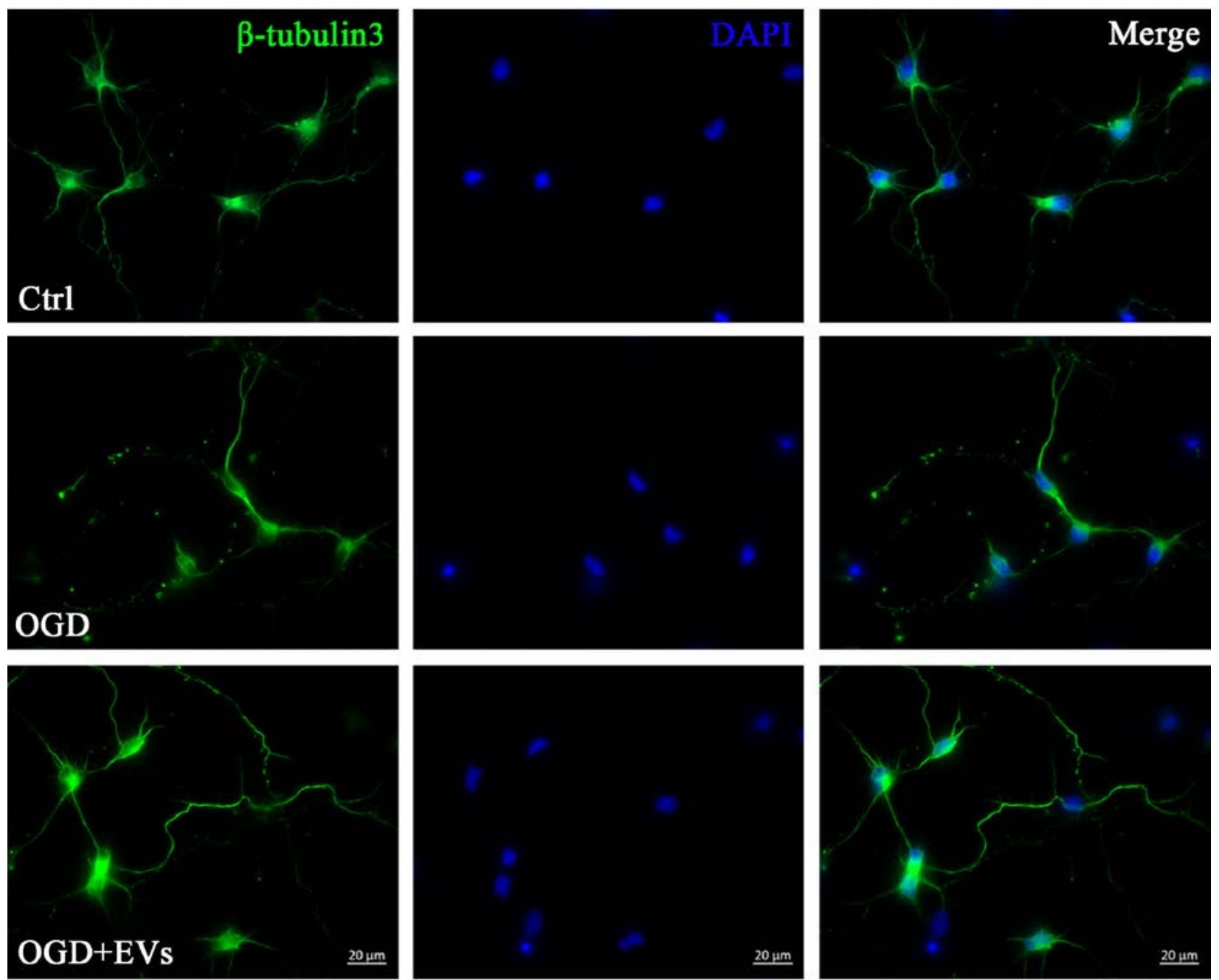

B

C

$\mathrm{D}$

$12 \mathrm{~h}$
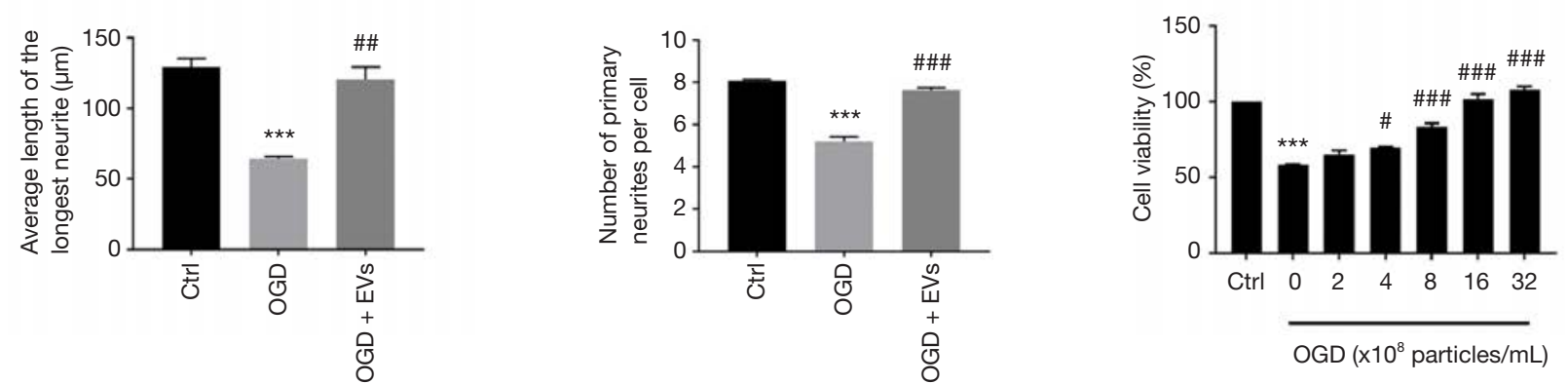

Figure 4 Reparative effect of SKP-SC-EVs on OGD-injured motoneurons. (A) Representative images of TUJ1 immunofluorescence stained motoneurons (green) in each group (control, OGD, OGD + EV) with blue DAPI labeled nuclei. Scale bar, $20 \mu$ m. (B,C) Statistical histograms of the average longest neurite length and the number of primary neurites per cell was better in OGD + EV group than that in OGD group. For each group and experiment, about 100 neurons per condition were assessed. (D) Statistical histograms showed the increasing neuronal cell viability in a dose-dependent manner. Mean $\pm \mathrm{SEM}, \mathrm{n}=3$; ${ }^{* *}, \mathrm{P}<0.001$, as compared with control group; ${ }^{*}$, $<<0.05$, \#\# $\mathrm{P}<0.01$, \#\#, $\mathrm{P}<0.001$, as compared with OGD group. SKP, skin precursor; SC, Schwann cell; EV, extracellular vesicle; OGD, oxygen glucose deprivation; TUJ1, $\beta$-tubulin3; DAPI, 4',6-diamidino-2-phenylindole. 
A

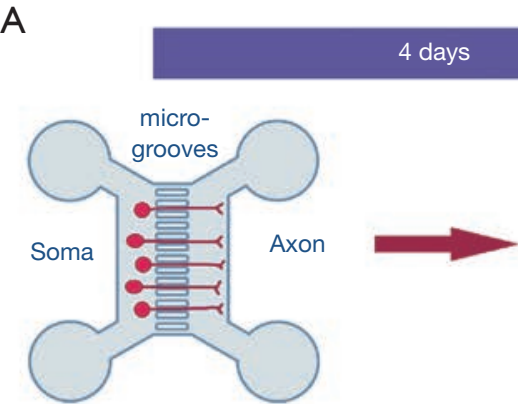

PRE-AXOTOMY

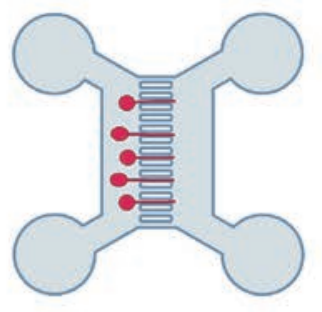

AXOTOMY
24 hours

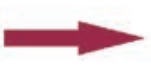

C
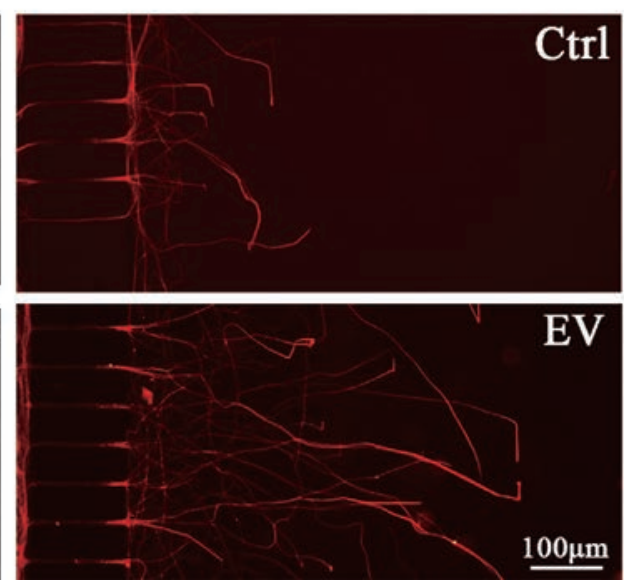

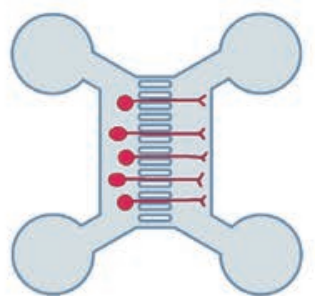

REGENERATION
B
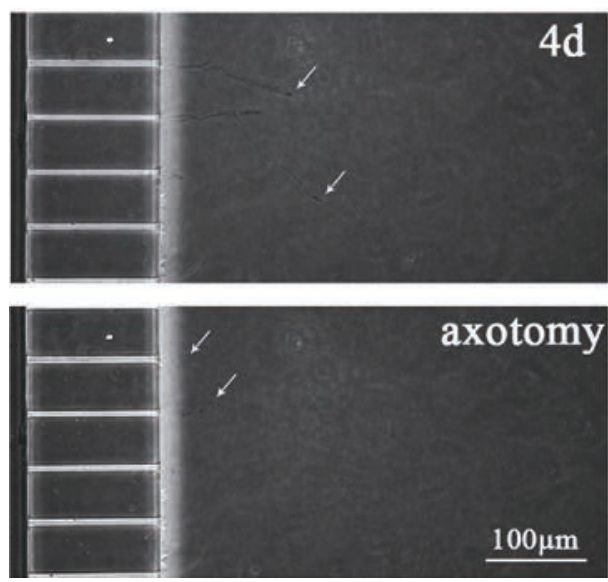

Figure 5 Axonal regrowth promoting effect of SKP-SC-EVs on axotomizing injury of motoneurons. (A) Schematic of axon outgrowth, axotomy, and axon regrowth of motoneurons in a microfluidic device, motoneurons were loaded into the soma chamber, then axons traversed through the microgrooves, at day 4 after culture the sprouting axons were transected by vacuum pump, followed by axonal regrowth into the axon chamber after 24 h. (B) Phase-contrast images of axons were displayed before (up; arrows) and after (bottom; arrows) axotomy, with the arrows on top panel indicating axon outgrowth, and the arrows on bottom panel indicating axon retraction by axotomy. Scale bar, $100 \mu \mathrm{m}$. (C) Representative image of regenerated axons (red) of TUJ1 immunofluorescence stained motoneurons in control and EV groups. Scale bar, $100 \mu \mathrm{m}$. (D) The histograms showed the average quantification of the 15 longest axonal lengths of control and EV groups. Mean \pm SEM, $\mathrm{n}=3,{ }^{* *}, \mathrm{P}<0.01$, as compared with control group. SKP, skin precursor; SC, Schwann cell; EV, extracellular vesicle; TUJ1, $\beta$-tubulin3.

manufacturability (25). Here, rat SKP-SCs were successfully induced and separated, with favorable proliferating capacity and stability for several passages, and the EVs release was promoted by culture in FBS-free proliferation medium for up to 2 days, which was a compelling foundation for applying the cell-free therapy to motoneuron damage. Our results demonstrated that SKP-SC-EVs exerted the desirable effect of promoting axonal-outgrowth or regrowth on motoneurons with or without OGD exposure with increasing cell viability. Furthermore, the therapeutic effect of pro-axonal regrowth on axotomized motoneurons with treatment of SKP-SC-EVs was verified both in vitro and in vivo. Moreover, the survival and recovery of damaged motoneurons might be attributed to the Akt/ mTOR/p70S6K signaling pathway activation mediated by SKP-SC-EVs. In agreement with these findings, the latest observation reported that SKP-SCs can yield better locomotor outcomes, and mitigate pathological thickening of the bladder wall in chronic SCI, the transplanted SKPSCs reduced the formation of glial scarring, and greatly strengthened the presence of endogenous SCs, myelinating thousands of sprouting/spared host axons in and around the injury site (26).

The first prerequisite for SKP-SC-EVs to exert 

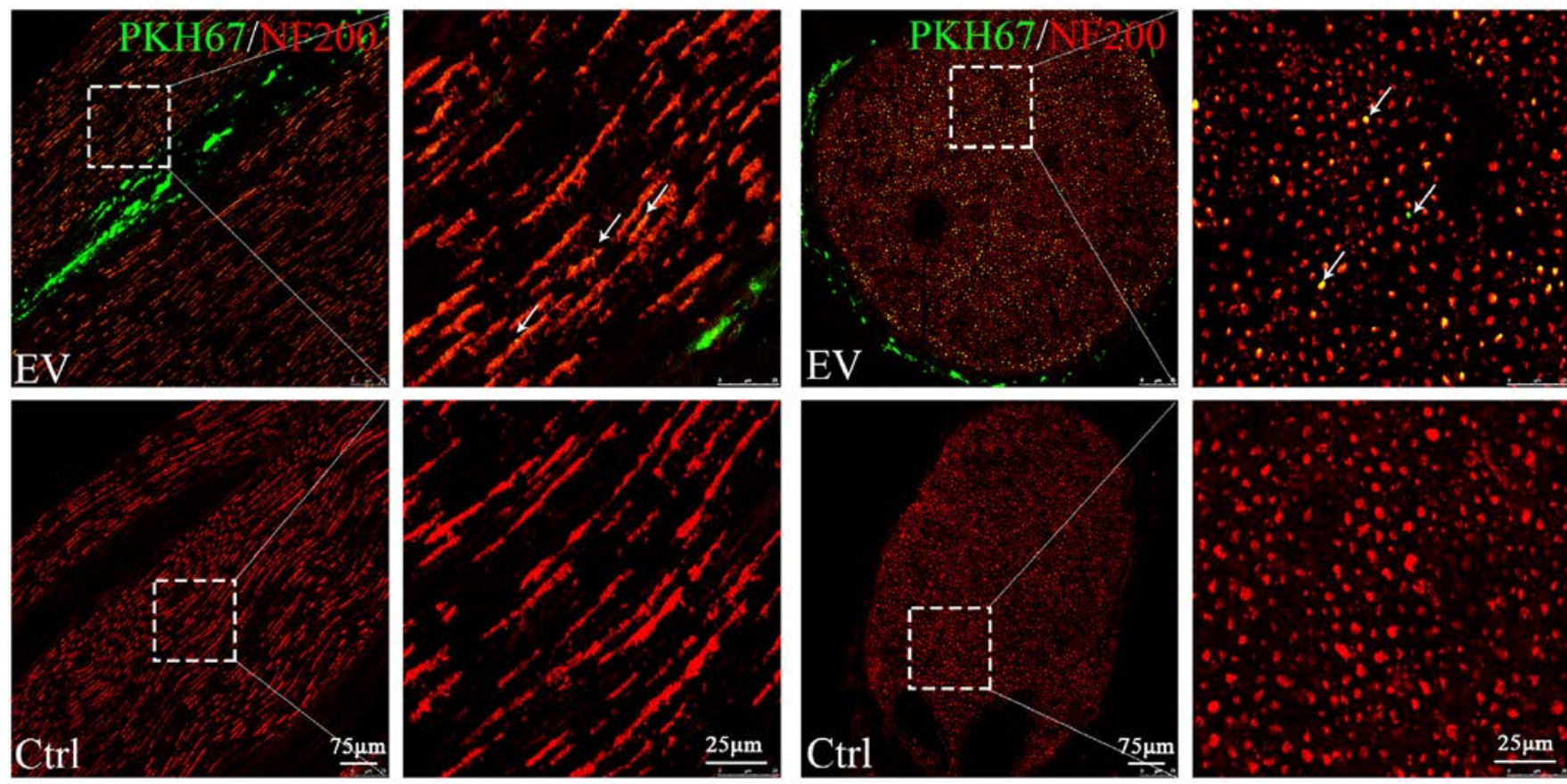

B
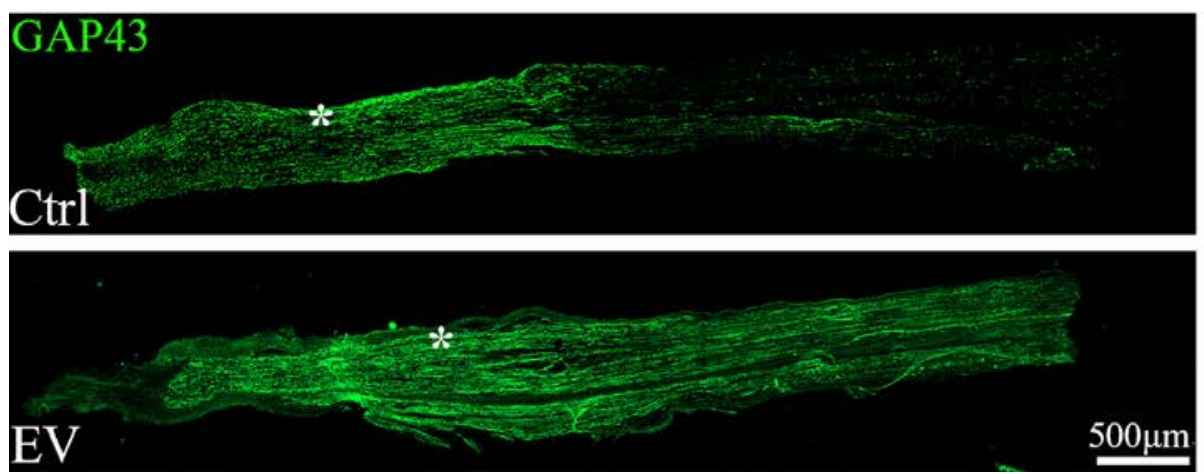

C

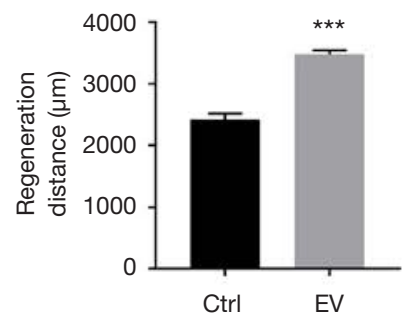

Figure 6 Pro-regenerative effect of SKP-SC-EVs on a crushed nerve. (A) PKH67-labeled EVs (green) were locally injected into intact sciatic nerves. SKP-SC-EVs were co-localized with neurofilament (NF200, red) in the longitudinal and cross sections of the nerve tissue. Scale bar, $75 \mu \mathrm{m}$. Right panels correspond to higher magnification of the boxed areas. Scale bar, $25 \mu \mathrm{m}$. (B) Axonal regeneration of sciatic nerve with injection of EVs or PBS (control) in vivo. GAP43 (green) stained regenerating axons (from left to right) showed longer extensions in EVs group than that in control group. The proximal stump of the crush site was indicated by the asterisk. Scale bar, $500 \mu \mathrm{m}$. (C) Statistical histograms showed the average distances distal from the lesion site of the GAP43-positive nerve fibers $4 \mathrm{~d}$ after crush injury. Mean \pm SEM, $\mathrm{n}=3$, ${ }^{* * *}, \mathrm{P}<0.001$, as compared with control group. SKP, skin precursor; SC, Schwann cell; EV, extracellular vesicle.

intercellular communication function was the internalization of EVs by recipient cells. Here, the intake of PKH67-labeled SKP-SC-EVs was displayed positioned both in soma and axon sites of primarily cultured motoneurons. Theoretically, the successful transfer of EVs into target cells would provide RNA, protein, and lipids (27), although the information reservoir of SKP-SC-EVs has not yet been identified. In addition, in this work, the EVs intake was also apparently displayed in the sciatic nerves after EVs microinjection locally, as we were aware that 
A

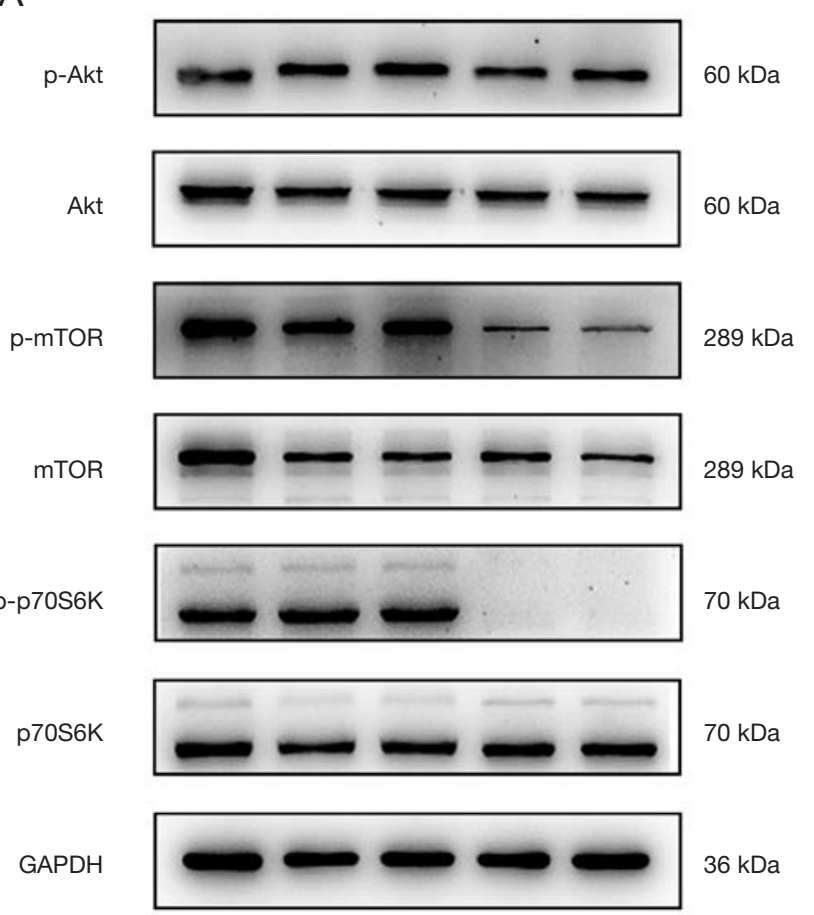

$\begin{array}{cccccc}\mathrm{OGD} & - & + & + & + & + \\ \mathrm{EV} & - & - & + & + & - \\ \text { Rapamycin } & - & - & - & + & +\end{array}$

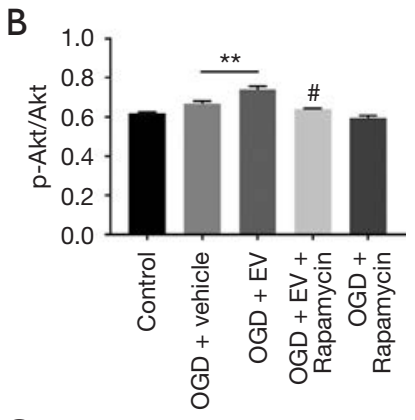

C
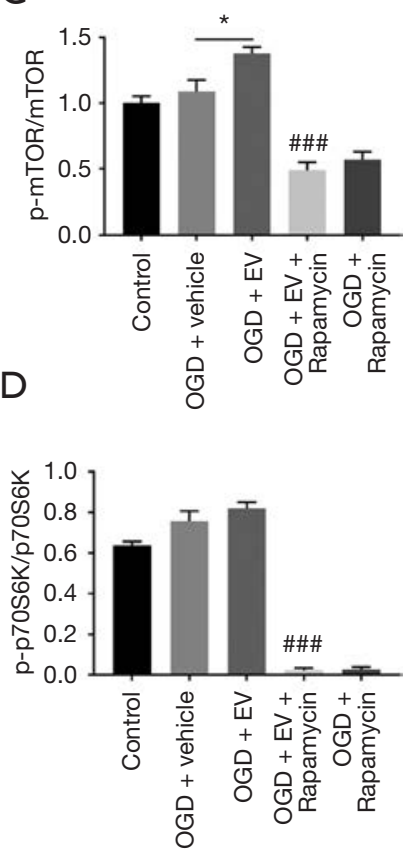

Figure 7 SKP-SC-EVs repair OGD-injured motoneurons by mTOR pathway activation. (A) Representative images of western blot analysis for p-Akt, Akt, p-mTOR, mTOR, p-p70S6K, and p70S6K expression of motoneurons in different groups. (B,C,D) Statistic histograms showed the increased expression level of p-Akt, p-mTOR, and p-p70S6K in OGD and OGD + EV group than in control group, and the significantly higher expression of p-Akt and p-mTOR in OGD + EV group than in OGD + vehicle group, while the signaling molecules expression was significantly abolished by rapamycin (an mTOR inhibitor). Mean $\pm \mathrm{SEM}, \mathrm{n}=3 ;{ }^{*}, \mathrm{P}<0.05,{ }^{* *}, \mathrm{P}<0.01$, as compared with $\mathrm{OGD}$ group; ${ }^{\#}, \mathrm{P}<0.05$, \#\#, $\mathrm{P}<0.001$, as compared with OGD + EV group. SKP, skin precursor; SC, Schwann cell; EV, extracellular vesicle; OGD, oxygen glucose deprivation.

local microinjection at the nerve epineurium site would ensure the intake of EVs by neural cells in nerve milieu, as the biodistribution of $\mathrm{EVs}$ is influenced by different routes administration (28). These results indicated that SKP-SCEVs can be effectively delivered to neural cells in vitro and in vivo.

Besides observing the neuroprotective effect of SKP-SC$\mathrm{EVs}$ on promoting the axonal outgrowth of motoneurons in a physiological situation, in order to further investigate their effect on damaged motoneurons, we constructed several neuronal injury models. To date, microfluidic devices with vacuum axotomy design and hypoxia incubators with OGD culture have been commonly utilized in vitro to simulate the neuronal injury in vivo. Firstly, OGD exposure in vitro was utilized to copy the secondary injury of motoneurons caused by ischemia and hypoxia in SCI. After treatment with EVs, results showed that SKP-SC-EVs could augment neuronal cell viability in a dose-dependent manner, and 
promote axonal regrowth significantly. Therefore, SKP-SCEVs can affect motoneurons in a dose-dependent manner in both physiological and pathological conditions. These data demonstrated the reparative potential of SKP-SC-EVs on the secondary ischemic hypoxic injury of motoneurons initiated by primary traumatic injury. There was good agreement with the effect of EVs from ADSC-derived SCs on promoting neurite outgrowth of neurons (16).

Correspondently, in this work, a unique platform utilizing a microfluidic device specifically enabled in vitro investigation of the effect of SKP-SC-EVs on axonal outgrowth and regrowth. Prospectively, the pro-axonal outgrowth effect of SKP-SC-EVs was effectively replicated in motoneurons cultured in chambers of the microfluidic device, with or without axotomizing injury. We added EVs to the soma side, axon side, and both sides respectively; results showed that supplementation of EVs to the axon side was more effective than supplementing the soma side, and there was no significant difference in supplementing both sides compared to just supplementing the axon side (data not shown). These findings suggested that axonal disruption could be directly ameliorated by the intake of SKP-SC-EVs at the axon lesion site, and the process might be independent of neuronal soma. This supported previous findings in literature that the uptake and retrograde transport of bone marrow MSC-derived exosomes can occur at peripheral nerve endings (29). Additionally, transection of the sciatic nerve due to crush injury in rats was effectively repaired by treatment with SKP-SC-EVs in our study; the effective regrowth of nerve fibers after mechanical injury in vitro and in vivo implied a pronounced therapeutic potential of SKP-SC-EVs for neuroregeneration. Our results are similar to the finding of $\mathrm{Ma}$ et al., that EVs from human umbilical cord MSCs can improve nerve regeneration after sciatic nerve transection in rats (30). Since the diversity of $\mathrm{EV}$ biogenesis mechanisms and the complexity of EV cargo content, EVs from different cell sources may have different functional principles, that remained be further investigated. To further verify the therapeutic potential of SKP-SC-EVs, we should apply them to rat nerve defect injury and SCI model in the future, which would enable more behavioral function and safety index tests to be performed.

Finally, we performed further examination on the classic pro-growth and pro-viability signaling pathway. Our results revealed a significantly elevated expression of p-Akt and p-mTOR signaling molecules after SKP-SC-EVs treatment on OGD-injured motoneurons, whereas the inhibition of
mTOR abrogated the activation of the Akt/mTOR/p70S6K signaling pathway. The activation of mTOR activation can be either Akt dependent or Akt independent. In previous work, the secretome or conditioned culture medium of SKP-SCs showed a protective effect on an in vitro Parkinson's disease model, which prevented SH-SY5Y cells from apoptosis against neurotoxicity by the PI3K/ Akt/Bcl-2 pathway (31). Additionally, ATP-mediated Akt/ mTOR/p70S6K pathway activation can promote the improvement of locomotor function after SCI in rats (32); furthermore, MSC derived EVs can increase the ATP levels and cell viability of damaged cells, reduce oxidative stress, and activate the PI3K/Akt pathway to alleviate the severity of cell injury (33). Moreover, resveratrol-primed exosomes can strongly promote the recovery of motor function in SCI rats by activating autophagy and inhibiting apoptosis via the PI3K/Akt/mTOR signaling pathway (34). However, the appropriate normalization strategy to compare quantitatively the functions of different EVs with drug therapy remains to be explored in future work because of the complexity of EV cargo content. In both secretome and EVs paracrine mediator models, the activation of Akt pathway and downstream signaling molecules seemed to have evoked endogenous neuroreparative mechanisms in a pathophysiological environment, which contrasts with the ability of the mTOR pathway to be Akt independent (35). Sestrin2 for example, also known as hypoxia inducible gene 95 , has emerged as a critical determinant of cell homeostasis in response to cellular stress, with the ability to attenuate endoplasmic reticulum stress and improve functional recovery by activating autophagy after SCI, and is dependent on the AMP-activated protein kinase (AMPK)/ mTOR/p70S6K pathway (36). Fibroblast-derived exosomes can mobilize an autocrine Wnt10b/mTOR pathway, thereby awakening the intrinsic capacity of neurons for regeneration (37). Suggestively, it seemed that SKP-SCsEVs could induce neurological recovery by a combination of different mechanisms via mTOR pathway activation, involving the promotion of neurogenesis, neuroprotection, autophagy/apoptosis regulation, and antioxidant defense. All these published observations further highlighted the significance of uncovering molecular mechanisms in our subsequent work.

It has been realized that maintaining neural microenvironment homeostasis is critical to sustain neuronal survival and axonal outgrowth (38). Various types of cells in neural tissue participate in the regulation 
of milieu homeostasis through paracrine communication; meanwhile, neurons also require their own homeostasis, including regulation of autophagy/apoptosis, that can be mediated by the Akt-dependent and Akt -independent mTOR pathway. Thus, it is a promising method to activate endogenous neuroprotection mechanisms for tissue regeneration by constructing a beneficial microenvironment via a system for delivering EVs.

In order to repair motoneuron impairment in traumatic injury of the brain, nerve, or spinal cord, and even prevent or reverse the neurological deficit in amyotrophic lateral sclerosis (ALS)/motoneuron disease, developing effective strategies based on an EVs platform is a valuable work. Intriguingly, exosomes from ADSCs can recover coupling efficiency and mitochondrial membrane potential in an in vitro model of ALS (39), and ameliorate the disease progression in SOD1 (G93A) a murine model (40), this work underlined the potential for therapeutic use of EVs in human ALS. Crucially, the identification of RNAs present in SC-derived EVs is a valuable avenue for understanding novel roles of SCs in neuronal function in health and disease (41). It was reported that EVs containing microRNA-29b (42), microRNA-25 (43), or microRNA-21-5p (44,45) can mediate neuroprotective effects and alleviate neural injury. Moreover, SC-derived exosomes were identified to enrich with proteins, among them 12 proteins are closely related to nervous system repair, involving different potential mechanisms, especially axon regeneration and inflammation inhibition; besides, through biological pathway analysis on the 12 proteins, it was showed that pathways are mostly involved in exosome biogenesis, formation, uptake and axon regeneration; among the pathways, the PI3KAkt, neurotrophin and cAMP signaling pathways played important roles in central nervous system repair (46). Compared to cell-based therapy, that must consider cell source, phenotype, number, delivery method, and immune rejection, the cell-free therapy of EVs intends to surmount these issues in future with bioengineered improvements to the above-mentioned features (47). The therapeutic applications of native and bioengineered EVs to neurological diseases would be expected to increase accordingly (48).

\section{Conclusions}

In summary, we utilized OGD-injury and axotomizing injury models to simulate the pathophysiological status of damaged motoneurons. Prospectively, SKP-SC-EVs showed desirable facilitation to bolstering the cell viability and axonal outgrowth of motoneurons. These basic findings implied the therapeutic potential of SKP-SC-EVs on PNI, SCI, and even ALS/motoneuron disease, and the activation of Akt-dependent or Akt-independent mTOR/p70S6K signaling pathway proposed an interesting molecular mechanism. This work established an experimental basis to explore elaborate mechanisms and develop preclinical translational trials of SKP-SC-EVs on neurological recovery.

\section{Acknowledgments}

We thank the reviewers and editors for their helpful comments on this article.

Funding: This work was supported by the National Key Technologies Research and Development Program of China (2017YFA0104700), National Natural Science Foundation of China (NSFC; 31870977), and Jiangsu Provincial Key Medical Center of China.

\section{Footnote}

Reporting Checklist: The authors have completed the ARRIVE reporting checklist. Available at http://dx.doi. org/10.21037/atm-20-5965

Data Sharing Statement: Available at http://dx.doi. org/10.21037/atm-20-5965

Conflicts of Interest: All authors have completed the ICMJE uniform disclosure form (available at http://dx.doi. org/10.21037/atm-20-5965). The authors have no conflicts of interest to declare.

Ethical Statement: The authors are accountable for all aspects of the work in ensuring that questions related to the accuracy or integrity of any part of the work are appropriately investigated and resolved. The study was approved by the Administration Committee of Experimental Animals, Jiangsu Province, China. The study protocols involving animals were performed according to the Chinese Guidelines for the Care and Use of Laboratory Animals.

Open Access Statement: This is an Open Access article 
distributed in accordance with the Creative Commons Attribution-NonCommercial-NoDerivs 4.0 International License (CC BY-NC-ND 4.0), which permits the noncommercial replication and distribution of the article with the strict proviso that no changes or edits are made and the original work is properly cited (including links to both the formal publication through the relevant DOI and the license). See: https://creativecommons.org/licenses/by-nc-nd/4.0/.

\section{References}

1. Wiklander OPB, Brennan MA, Lotvall J, et al. Advances in therapeutic applications of extracellular vesicles. Sci Transl Med 2019;11:eaav8521.

2. GBD 2016 Traumatic Brain Injury and Spinal Cord Injury Collaborators. Global, regional, and national burden of traumatic brain injury and spinal cord injury, 1990-2016: a systematic analysis for the Global Burden of Disease Study 2016. Lancet Neurol 2019;18:56-87.

3. EL Andaloussi S, Mäger I, Breakefield XO, et al. Extracellular vesicles: biology and emerging therapeutic opportunities. Nat Rev Drug Discov 2013;12:347-57.

4. Pick H, Alves AC, Vogel H. Single-Vesicle Assays Using Liposomes and Cell-Derived Vesicles: From Modeling Complex Membrane Processes to Synthetic Biology and Biomedical Applications. Chem Rev 2018;118:8598-654.

5. van der Pol E, Boing AN, Harrison P, et al. Classification, functions, and clinical relevance of extracellular vesicles. Pharmacol Rev 2012;64:676-705.

6. Kim SY, Khanal D, Tharkar P, et al. None of us is the same as all of us: resolving the heterogeneity of extracellular vesicles using single-vesicle, nanoscale characterization with resonance enhanced atomic force microscope infrared spectroscopy (AFM-IR). Nanoscale Horiz 2018;3:430-8.

7. Witwer KW, Van Balkom BWM, Bruno S, et al. Defining mesenchymal stromal cell (MSC)-derived small extracellular vesicles for therapeutic applications. J Extracell Vesicles 2019;8:1609206.

8. Maqsood M, Kang M, Wu X, et al. Adult mesenchymal stem cells and their exosomes: Sources, characteristics, and application in regenerative medicine. Life Sci 2020;256:118002.

9. Min Q, Parkinson DB, Dun XP. Migrating Schwann cells direct axon regeneration within the peripheral nerve bridge. Glia 2021;69:235-54.

10. Madison RD, Robinson GA. Muscle-Derived Extracellular Vesicles Influence Motor Neuron Regeneration Accuracy.
Neuroscience 2019;419:46-59.

11. Wang J, Rong Y, Ji C, et al. MicroRNA-421-3p-abundant small extracellular vesicles derived from M2 bone marrowderived macrophages attenuate apoptosis and promote motor function recovery via inhibition of mTOR in spinal cord injury. J Nanobiotechnology 2020;18:72 .

12. Mehrotra P, Tseropoulos G, Bronner ME, et al. Adult tissue-derived neural crest-like stem cells: Sources, regulatory networks, and translational potential. Stem Cells Transl Med 2020;9:328-41.

13. Jing $\mathrm{H}, \mathrm{He} \mathrm{X}$, Zheng J. Exosomes and regenerative medicine: state of the art and perspectives. Transl Res 2018;196:1-16.

14. Giebel B, Hermann DM. Identification of the right cell sources for the production of therapeutically active extracellular vesicles in ischemic stroke. Ann Transl Med 2019;7:188.

15. Lopez-Verrilli MA, Picou F, Court FA. Schwann cellderived exosomes enhance axonal regeneration in the peripheral nervous system. Glia 2013;61:1795-806.

16. Ching RC, Wiberg M, Kingham PJ. Schwann celllike differentiated adipose stem cells promote neurite outgrowth via secreted exosomes and RNA transfer. Stem Cell Res Ther 2018;9:266.

17. Khuong HT, Kumar R, Senjaya F, et al. Skin derived precursor Schwann cells improve behavioral recovery for acute and delayed nerve repair. Exp Neurol 2014;254:168-79.

18. Sparling JS, Bretzner F, Biernaskie J, et al. Schwann cells generated from neonatal skin-derived precursors or neonatal peripheral nerve improve functional recovery after acute transplantation into the partially injured cervical spinal cord of the rat. J Neurosci 2015;35:6714-30.

19. McDonald JW, Sadowsky C. Spinal-cord injury. Lancet 2002;359:417-25.

20. Ahuja CS, Martin AR, Fehlings M. Recent advances in managing a spinal cord injury secondary to trauma. F1000Res 2016;5:F1000 Faculty Rev-1017.

21. Ahuja CS, Nori S, Tetreault L, et al. Traumatic Spinal Cord Injury-Repair and Regeneration. Neurosurgery 2017;80:S9-22.

22. Biernaskie JA, McKenzie IA, Toma JG, et al. Isolation of skin-derived precursors (SKPs) and differentiation and enrichment of their Schwann cell progeny. Nat Protoc 2006;1:2803-12.

23. Haastert K, Grosskreutz J, Jaeckel M, et al. Rat embryonic motoneurons in long-term co-culture with Schwann cells- 
-a system to investigate motoneuron diseases on a cellular level in vitro. J Neurosci Methods 2005;142:275-84.

24. Taylor AM, Blurton-Jones M, Rhee SW, et al. A microfluidic culture platform for CNS axonal injury, regeneration and transport. Nat Methods 2005;2:599-605.

25. Reiner AT, Witwer KW, van Balkom BWM, et al. Concise Review: Developing Best-Practice Models for the Therapeutic Use of Extracellular Vesicles. Stem Cells Transl Med 2017;6:1730-9.

26. Assinck P, Sparling JS, Dworski S, et al. Transplantation of Skin Precursor-Derived Schwann Cells Yields Better Locomotor Outcomes and Reduces Bladder Pathology in Rats with Chronic Spinal Cord Injury. Stem Cell Reports 2020;15:140-55.

27. Skotland T, Sagini K, Sandvig K, et al. An emerging focus on lipids in extracellular vesicles. Adv Drug Deliv Rev 2020;S0169-409X(20)30014-4.

28. Wiklander OP, Nordin JZ, O'Loughlin A, et al. Extracellular vesicle in vivo biodistribution is determined by cell source, route of administration and targeting. J Extracell Vesicles 2015;4:26316.

29. Ren R, Tan XH, Zhao JH, et al. Bone marrow mesenchymal stem cell-derived exosome uptake and retrograde transport can occur at peripheral nerve endings. Artif Cells Nanomed Biotechnol 2019;47:2918-29.

30. Ma Y, Dong L, Zhou D, et al. Extracellular vesicles from human umbilical cord mesenchymal stem cells improve nerve regeneration after sciatic nerve transection in rats. J Cell Mol Med 2019;23:2822-35.

31. Chen Y, Shen J, Ma C, et al. Skin-derived precursor Schwann cells protect SH-SY5Y cells against 6-OHDAinduced neurotoxicity by PI3K/AKT/Bcl-2 pathway. Brain Res Bull 2020;161:84-93.

32. Hu LY, Sun ZG, Wen YM, et al. ATP-mediated protein kinase B Akt/mammalian target of rapamycin mTOR/ p70 ribosomal S6 protein p70S6 kinase signaling pathway activation promotes improvement of locomotor function after spinal cord injury in rats. Neuroscience 2010;169:1046-62.

33. Arslan F, Lai RC, Smeets MB, et al. Mesenchymal stem cell-derived exosomes increase ATP levels, decrease oxidative stress and activate PI3K/Akt pathway to enhance myocardial viability and prevent adverse remodeling after myocardial ischemia/reperfusion injury. Stem Cell Res 2013;10:301-12.

34. Fan Y, Li Y, Huang S, et al. Resveratrol-primed exosomes strongly promote the recovery of motor function in SCI rats by activating autophagy and inhibiting apoptosis via the PI3K signaling pathway. Neurosci Lett 2020;736:135262.

35. Hsu JL, Leu WJ, Hsu LC, et al. Para-Toluenesulfonamide Induces Anti-tumor Activity Through Akt-Dependent and -Independent mTOR/p70S6K Pathway: Roles of Lipid Raft and Cholesterol Contents. Front Pharmacol 2018;9:1223.

36. Li Y, Zhang J, Zhou K, et al. Elevating sestrin2 attenuates endoplasmic reticulum stress and improves functional recovery through autophagy activation after spinal cord injury. Cell Biol Toxicol 2020. [Epub ahead of print]. doi: 10.1007/s10565-020-09550-4.

37. Tassew NG, Charish J, Shabanzadeh AP, et al. Exosomes Mediate Mobilization of Autocrine Wnt10b to Promote Axonal Regeneration in the Injured CNS. Cell Rep 2017;20:99-111.

38. Fan B, Wei Z, Yao X, et al. Microenvironment Imbalance of Spinal Cord Injury. Cell Transplant 2018;27:853-66.

39. Calabria E, Scambi I, Bonafede R, et al. ASCs-Exosomes Recover Coupling Efficiency and Mitochondrial Membrane Potential in an in vitro Model of ALS. Front Neurosci 2019;13:1070.

40. Bonafede R, Turano E, Scambi I, et al. ASC-Exosomes Ameliorate the Disease Progression in SOD1(G93A) Murine Model Underlining Their Potential Therapeutic Use in Human ALS. Int J Mol Sci 2020;21:3651.

41. De Gregorio C, Diaz P, Lopez-Leal R, et al. Purification of Exosomes from Primary Schwann Cells, RNA Extraction, and Next-Generation Sequencing of Exosomal RNAs. Methods Mol Biol 2018;1739:299-315.

42. Yu T, Zhao C, Hou S, et al. Exosomes secreted from miRNA-29b-modified mesenchymal stem cells repaired spinal cord injury in rats. Braz J Med Biol Res 2019;52:e8735.

43. Zhao L, Jiang X, Shi J, et al. Exosomes derived from bone marrow mesenchymal stem cells overexpressing microRNA-25 protect spinal cords against transient ischemia. J Thorac Cardiovasc Surg 2019;157:508-17.

44. Zhou X, Chu X, Yuan H, et al. Mesenchymal stem cell derived EVs mediate neuroprotection after spinal cord injury in rats via the microRNA-21-5p/FasL gene axis. Biomed Pharmacother 2019;115:108818.

45. Gao X, Xiong Y, Li Q, et al. Extracellular vesicle-mediated transfer of miR-21-5p from mesenchymal stromal cells to neurons alleviates early brain injury to improve cognitive function via the PTEN/Akt pathway after subarachnoid 
hemorrhage. Cell Death Dis 2020;11:363.

46. Wei Z, Fan B, Ding H, et al. Proteomics analysis of Schwann cell-derived exosomes: a novel therapeutic strategy for central nervous system injury. Mol Cell Biochem 2019;457:51-9.

47. Huang YC, Lai LC. The potential roles of stem cell- derived extracellular vesicles as a therapeutic tool. Ann Transl Med 2019;7:693.

48. de Abreu RC, Fernandes H, da Costa Martins PA, et al. Native and bioengineered extracellular vesicles for cardiovascular therapeutics. Nat Rev Cardiol 2020;17:685-97.
Cite this article as: Wu X, Wang L, Cong M, Shen M, He Q, Ding F, Shi H. Extracellular vesicles from skin precursorderived Schwann cells promote axonal outgrowth and regeneration of motoneurons via $\mathrm{Akt} / \mathrm{mTOR} / \mathrm{p} 70 \mathrm{~S} 6 \mathrm{~K}$ pathway. Ann Transl Med 2020;8(24):1640. doi: 10.21037/atm-20-5965 